\title{
Time scale and other invariants of integrative mechanical behavior in living cells
}

\author{
Ben Fabry, ${ }^{1, *}$ Geoffrey N. Maksym, ${ }^{2}$ James P. Butler, ${ }^{1}$ Michael Glogauer, ${ }^{3}$ Daniel Navajas, ${ }^{4}$ Nathan A. Taback,${ }^{5}$ \\ Emil J. Millet, ${ }^{1}$ and Jeffrey J. Fredberg ${ }^{1}$ \\ ${ }^{1}$ Physiology Program, Harvard School of Public Health, 665 Huntington Avenue, Boston, Massachusetts 02115, USA \\ ${ }^{2}$ School of Biomedical Engineering, Dalhousie University, 5981 University Avenue, Halifax, Nova Scotia, Canada B3H $3 J 5$ \\ ${ }^{3}$ Faculty of Dentistry, University of Toronto, 150 College Street, Toronto, Ontario, Canada M5S $3 E 2$ \\ ${ }^{4}$ Unitat Biofísica i Bioenginyeria, Universitat de Barcelona-IDIBAPS, Casanova 143, 08036 Barcelona, Spain \\ ${ }^{5}$ Department of Biostatistics, Harvard School of Public Health, 665 Huntington Avenue, Boston, Massachusetts 02115, USA
}

(Received 7 May 2003; published 27 October 2003)

\begin{abstract}
In dealing with systems as complex as the cytoskeleton, we need organizing principles or, short of that, an empirical framework into which these systems fit. We report here unexpected invariants of cytoskeletal behavior that comprise such an empirical framework. We measured elastic and frictional moduli of a variety of cell types over a wide range of time scales and using a variety of biological interventions. In all instances elastic stresses dominated at frequencies below $300 \mathrm{~Hz}$, increased only weakly with frequency, and followed a power law; no characteristic time scale was evident. Frictional stresses paralleled the elastic behavior at frequencies below $10 \mathrm{~Hz}$ but approached a Newtonian viscous behavior at higher frequencies. Surprisingly, all data could be collapsed onto master curves, the existence of which implies that elastic and frictional stresses share a common underlying mechanism. Taken together, these findings define an unanticipated integrative framework for studying protein interactions within the complex microenvironment of the cell body, and appear to set limits on what can be predicted about integrated mechanical behavior of the matrix based solely on cytoskeletal constituents considered in isolation. Moreover, these observations are consistent with the hypothesis that the cytoskeleton of the living cell behaves as a soft glassy material, wherein cytoskeletal proteins modulate cell mechanical properties mainly by changing an effective temperature of the cytoskeletal matrix. If so, then the effective temperature becomes an easily quantified determinant of the ability of the cytoskeleton to deform, flow, and reorganize.
\end{abstract}

DOI: 10.1103/PhysRevE.68.041914

PACS number(s): 87.16.Ka, 83.85.Vb, 64.70.Pf, 87.19.Rr

\section{INTRODUCTION}

In response to the application of a mechanical stress, the cytoskeleton has the ability to deform, flow, and remodel. In the living cell, these processes involve the coordinated assembly and disassembly of cytoskeletal polymers as well as the coupling of those structures to motor proteins. Deformation, flow, and remodeling are fundamental to a variety of higher cell functions including cell contraction, adhesion, spreading, crawling, invasion, wound healing, and division, and have been implicated as well in mechanotransduction, regulation of protein and DNA synthesis, and programmed cell death [1]. Our understanding of the empirical constitutive laws that govern these mechanical processes remains quite fragmentary, however, as does our understanding of underlying mechanism. The manner in which the multitude of cytoskeletal and associated proteins interact with one another within the dynamic microenvironment of the cell body so as to produce integrated mechanical effects remains a major open question.

Although mechanical behavior of the cell is important in its own right, it can also be seen as being only one instance within the broader rubric of integrative phenotypic phenomena. We demonstrate in this paper that microrheology of the living cell may provide a unique window on protein dynamics in complex functional systems, by which we mean those

*Email address: bfabry@hsph.harvard.edu dominated by multiple weak effects operating within a noisy microenvironment and far from thermodynamic equilibrium. We address three questions. First, what might comprise a favorable model system for the study of molecular events that underlie integrative system functions? Second, might there be generic phenotypic properties that are characteristic of such systems? Finally, are there fundamental principles of biological design that govern integrative system functions?

Within the living cell, the universe of protein interactions is populated unevenly, with a very few highly connected hubs and a great many less connected nodes [2]; indeed, the probability of any given protein having $k$ interactions decreases as a power law with $k$. This power-law dependence shows that the highly interconnected network of biological interactions possesses no internal scale that can typify the number of interactions per protein and, accordingly, in that regard biological networks are said to be scale-free [2]. In turn, individual proteins comprising the network have been shown to be characterized by a very special structure; the universe of protein structures is populated unevenly, with a very few clusters, folds, and superfold domains that are rather common, but a great many fold domains that are much less common [3]. Interestingly, as protein size increases, the probability of any given number of fold domains varies as a power law with protein size. This power-law dependence shows that protein structure possesses no internal scale that can typify the number of folds per protein. Like network topology, protein structure is also scale-free [3]. The spatial distribution of structure density within the cell has been mea- 
sured, and the limited data that are available suggest that it too shows a power-law distribution and, therefore, appears to be scale-free [4].

The topology of the protein interaction network, taken together with the structures of the individual proteins themselves and their distribution in space, comprises the stage upon which protein dynamics must be played out. We had set out to identify distinct internal time scales, relaxation times, or time constants that might typify mechanical responses of the integrated cytoskeletal matrix; in rheology, mechanical relaxation processes are taken as being the shadow of underlying molecular dynamics [5]. We report here that over a spectrum spanning five decades of frequency and in all of the five cell types that were investigated, relaxation times were found to be distributed as a power law, with a great many relaxation processes contributing when the time scale of the imposed forcing was long, but very few as the time scale of the forcing was reduced and slower processes became progressively frozen-out of the response. As regards protein interactions within the complex microenvironment of the living intact cytoskeleton, therefore, there was no internal time scale that could typify their dynamics. All time scales were present simultaneously but distributed very unevenly; the integrated mechanical behavior was scale-free. Scale-free behavior thus pertains at the levels of the topology of the protein-protein interaction network, the structure and distribution of the individual proteins, and the dynamics of the matrix that they comprise. As described below, this finding stands in contrast with current theories of cytoskeletal behavior, all of which posit behavior characterized by one or, at most, a few distinct internal time scales.

We found in addition that regardless of the frequency of the imposed oscillation, the cell type that was studied, the signal transduction pathway that was activated, or the type of molecule that was manipulated, all data could be collapsed onto master curves that depended on only a single parameter [6]. These master curves demonstrate that when the mechanical properties of the cell change, they must do so along a special trajectory.

These findings, taken together, establish unanticipated invariants of cytoskeletal mechanical behavior and, in doing so, would appear to set limits on what can be predicted about the integrated mechanical behavior of the cell from considerations of cytoskeletal molecules studied in isolation. In particular, they lead to the notion that the integrated mechanical behavior of the cytoskeleton may not depend so much on the molecular details of particular cytoskeletal elements but instead may depend more on the generic metastability of their microstructural arrangements, as described in greater detail below. For any such arrangement, an index of this metastability can be given as the level of mechanical agitation (noise) present in the microenvironment relative to the depth of the energy well that defines that arrangement. This index is easily measured and can be expressed as an effective temperature of the matrix. As explained below, behavior of this kind is consistent with the hypothesis that the cytoskeletal matrix of the living adherent cell behaves as a soft glassy material [7]; here and elsewhere in this paper we use the term "cytoskeleton" in the broadest sense so as to subsume scaffolding proteins, the contractile apparatus, and any attached structures or molecules that contribute appreciably to the integrated mechanical properties of the cell. This paper expands on our previous short communication [6] by providing greater methodological detail, expanded data sets, and new statistical analysis.

\section{METHODS}

To study the rheology of cytoskeletal polymers requires a probe whose operative frequency range spans, in so far as possible, the internal molecular time scales of the rate processes in question $[8,9]$. Here we used magnetic twisting cytometry (MTC) with optical detection of bead motion. With this method, we were able to apply probing frequencies ranging from $0.01 \mathrm{~Hz}$ to $1 \mathrm{kHz}[10-14]$.

Microbead preparation. As a measurement probe we used ferrimagnetic microbeads $(4.5 \pm 0.3 \mu \mathrm{m}$ diam $)$ that were bound to specific cell surface receptors. We produced the beads by aerosolizing a water-based, surfactant stabilized $\mathrm{Fe}_{3} \mathrm{O}_{4}$ ferrofluid (Ferrotec, Nashua, $\mathrm{NH}$ ) with a spinning top aerosol generator (BGI inc., Waltham, MA). The aerosolized particles were dried in a copper funnel, neutralized with a radioactive beta source (americium), and then sintered for several seconds at $800{ }^{\circ} \mathrm{C}$ while suspended in an atmosphere containing $2 \% \mathrm{H}_{2}$ and $98 \% \mathrm{~N}_{2}$ (see [15] for details). We coated the beads overnight or longer at $4{ }^{\circ} \mathrm{C}$ with a synthetic peptide that contained the sequence RGD (Arg-Gly-Asp) [16] (Peptite 2000, Integra Life Sciences, San Diego, kindly provided by Dr. Jürg Tschopp) at $50 \mu \mathrm{g}$ peptide per mg beads in $1 \mathrm{ml}$ carbonate buffer ( $p \mathrm{H}$ 9.4). These beads were used for all cell types except neutrophils. In experiments with neutrophils we used polystyrene beads (Spherotech, Libertyville, IL) that were coated with chromium dioxide particles and precoated with goat-anti-mouse immunoglobulin $\mathrm{G}$ as a primary antibody. We coated those beads with a secondary mouse-anti-human CD-45 antibody (BD PharMingen, San Diego, CA) overnight at $4{ }^{\circ} \mathrm{C}$ at a concentration of $200 \mu \mathrm{g}$ antibodies per $10^{8}$ beads in $1 \mathrm{ml}$ phosphatebuffered saline.

Bead twisting. After the beads were bound to the cells (described below), the beads were magnetized horizontally by a brief $(0.1 \mathrm{~ms})$ magnetic field pulse $(>0.1 \mathrm{~T})$ and then twisted vertically by an external homogeneous magnetic field $(<0.01 \mathrm{~T})$ that was varying sinusoidally in time [Fig. 1(c)]. The twisting field induces a mechanical torque that tends to align the magnetic orientation of the bead with that of the magnetic twisting field.

The specific torque $(T)$ is defined as the mechanical torque per unit bead volume, and has dimensions of stress (Pa). $T$ is given by

$$
T=c H \cos \theta,
$$

where $H$ is the magnetic twisting field (in gauss), $\theta$ is the angle of the bead's magnetic moment relative to the original magnetization direction, and $c$ is the bead constant, expressed as torque per unit bead volume per gauss. $c$ is determined by placing beads in a fluid of known viscosity and 

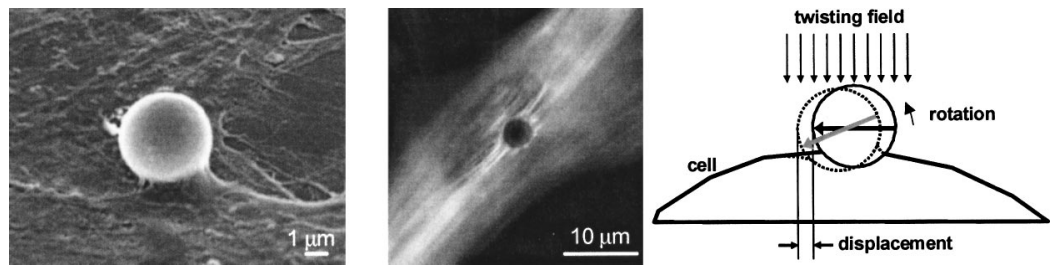

FIG. 1. (a) Scanning EM of a bead bound to the surface of a HASM cell. (b) Ferrimagnetic beads coated with RGD-containing peptide bind avidly to the actin cytoskeleton (stained with fluorescently labeled phalloidin) of HASM cells via cell adhesion molecules (integrins). (c) A magnetic twisting field introduces a torque which causes the bead to rotate and to displace. Large arrows indicate the direction of the bead's magnetic moment before (black) and after (gray) twisting. If the twisting field is varied sinusoidally in time, then the microbead wobbles to and fro, resulting in a lateral displacement, $d$, that can be measured.

then measuring the angular velocity while twisting [10]. In this study, we used beads with $c=1.86 \mathrm{~Pa} / \mathrm{G}$. Because $\theta$ was small [13], we could ignore the $\cos \theta$ term in Eq. (1) when computing the specific torque from the twisting field (see below). This resulted in errors of less than 5\% for small bead rotations $\left(\theta<18^{\circ}\right)$ or small lateral bead displacements $(<700 \mathrm{~nm}$ in the case of a pivoting motion of the bead around a contact point at the bead perimeter).

Measurement of bead motion. In response to a twisting field that varied sinusoidally in time, beads oscillated laterally to and fro, mainly along the magnetization direction. We eliminated beads from subsequent data analysis when their movement in the magnetization direction was smaller than their movement orthogonal to the magnetization direction; this was the case in about $10 \%$ of all beads. Lateral bead displacement, $d$, in response to the imposed oscillatory torque was observed with an inverted microscope (Diaphot, Nikon, Kanagawa, Japan) with a $10 \times$ objective (NA 0.2). The microscope was placed on a vibration isolation table (Newport, Irvine, CA). A progressive scan, triggerable blackand-white CCD camera with pixel-clock synchronization (JAI CV-M10, Glostrup, Denmark) was attached to the camera side-port of the microscope via a camera adapter with $1 \times$ magnification. Image acquisition (exposure time $100 \mu \mathrm{s}$ ) was phase-locked to the twisting field so that 16 images were acquired during a twisting cycle. Heterodyning (a stroboscopic technique) was used at frequencies $>1 \mathrm{~Hz}$. The phase separation between consecutive images was $\pi / 8$ when heterodyning was not used, or $2 \pi n+\pi / 8$ when heterodyning was used, with $n=1$ at frequencies of $4.5 \mathrm{~Hz}$ or $10 \mathrm{~Hz}, n$ $=3$ at $30 \mathrm{~Hz}, n=10$ at $100 \mathrm{~Hz}, n=30$ at $300 \mathrm{~Hz}$, and $n$ $=100$ at $1000 \mathrm{~Hz}$. The camera trigger signal and the signal for the twisting current driver were generated with a 16-bit microcontroller (C167, Infineon, Munich, Germany). The twisting current was generated with a current source capable of driving up to $2.5 \mathrm{~A}$. The images were analyzed on-line using an intensity-weighted center-of-mass algorithm for determining the bead position with an accuracy of $5 \mathrm{~nm}$ (rms) (see [14] for details).

Measurement of elastic moduli. As the bead moves, it deforms the cell to which it is bound [17]. The cell resists this bead motion by developing internal stresses that depend on the cell's mechanical properties. Thus, cell mechanical properties could be calculated from the imposed mechanical torque and the measurement of the resulting bead motion.

The ratio of the complex specific torque $\widetilde{T}$ to the resulting complex bead displacement $\widetilde{d}$ defines a complex elastic modulus of the cell $\widetilde{g}(f)=\widetilde{T}(f) / \widetilde{d}(f)$ as a function of frequency $f$, and has dimensions of $\mathrm{Pa} / \mathrm{nm}$. For each bead we computed the elastic modulus, or stiffness, $g^{\prime}$ (the real part of $\widetilde{g}$ ), the loss modulus, or friction, $g^{\prime \prime}$ (the imaginary part of $\widetilde{g}$ ), and the loss tangent, or hysteresivity, $\eta$ (the ratio $g^{\prime \prime} / g^{\prime}$ ). These measurements could be transformed into traditional elastic shear $\left(G^{\prime}\right)$ and loss $\left(G^{\prime \prime}\right)$ moduli by a geometric factor $\alpha$ that depends on the shape and thickness of the cell, and the degree of bead embedding, where $\widetilde{G}=\alpha \widetilde{g}, \widetilde{G}=G^{\prime}$ $+i G^{\prime \prime}$, and $i^{2}=-1$. Finite element analysis of cell deformation for a representative bead-cell geometry (assuming homogeneous and isotropic elastic properties with $10 \%$ of the bead diameter embedded in a cell $5 \mu \mathrm{m}$ high) sets $\alpha$ to 6.8 $\mu \mathrm{m}$ [17]. This geometric factor need serve only as a rough approximation, however, because it cancels out in the scaling procedure described below, which is model-independent.

To test the entire measurement system, we studied the frequency response of collagen-coated beads that were covalently crosslinked to the surface of a polyacrylamide gel ( $7 \%$ acrylamide with $0.25 \%$ bis-acrylamide as a crosslinker) with a low-frequency stiffness of $7 \mathrm{kPa}$ [18]. Our measurements indicated a predominantly elastic response of the gel, with a $g^{\prime}$ that was flat between $0.01 \mathrm{~Hz}$ and $1000 \mathrm{~Hz}$. This behavior closely resembled the behavior of an identically prepared polyacrylamide gel that we measured with diffusing-wave spectroscopy using the method of Mason and Weitz [19].

Data analysis. To describe our data, we used the structural damping law (sometimes called hysteretic damping) together with a small additive Newtonian viscosity term [20-25]. The mechanical properties of such a material can be mathematically expressed either in the time domain or in the frequency domain. In the time domain, the mechanical stress response to a unit step change in strain imposed at $t=0$ is an instantaneous component attributable to a pure viscous response together with a component that rises instantaneously and then decays over time as a power law,

$$
g(t)=\mu \delta(t)+g_{0}\left(t / t_{0}\right)^{1-x} .
$$

$g_{0}$ is the ratio of stress to the unit strain measured at an arbitrarily chosen time $t_{0}, \mu$ is a Newtonian viscous term, and $\delta$ is the Dirac delta. The stress response to unit amplitude sinusoidal deformations can be obtained by taking the Fou- 
rier transform of the step response [Eq. (2)] and multiplying by $i \omega$, which gives the complex modulus $\widetilde{g}(\omega)$ as

$$
\widetilde{g}(\omega)=g_{0}\left(\frac{\omega}{\Phi_{0}}\right)^{x-1}(1+i \bar{\eta}) \Gamma(2-x) \cos \frac{\pi}{2}(x-1)+i \omega \mu,
$$

where $\bar{\eta}=\tan (x-1) \pi / 2$ and $\omega$ is the radian frequency $2 \pi f$. $g_{0}$ and $\Phi_{0}$ are scale factors for stiffness and frequency, respectively, and $\Gamma$ denotes the gamma function. $g_{0}$ and $\mu$ depend on bead-cell geometry. $\bar{\eta}$ has been called the structural damping coefficient [25]. The elastic modulus $g^{\prime}$ corresponds to the real part of Eq. (3), which increases for all $\omega$ according to the power-law exponent, $x-1$. The loss modulus $g^{\prime \prime}$ corresponds to the imaginary part of Eq. (3) and includes a component which also increases as a power law with the same exponent. Therefore, this component is a frequency-independent fraction $(\bar{\eta})$ of the elastic modulus; such a direct coupling of the loss modulus to the elastic modulus is the characteristic feature of structural damping behavior [25]. The loss modulus also includes a Newtonian viscous term, $i \omega \mu$, which turns out to be small except at very high frequencies. At low frequencies, the loss tangent $\eta$ approximates $\bar{\eta}$. In the limit that $x$ approaches unity, the power-law slope approaches zero, $g^{\prime}$ approaches $g_{0}$, and $\bar{\eta}$ approaches zero. In the limit that $x$ approaches 2 , the powerlaw slope approaches unity, $g^{\prime \prime}$ approaches $\mu$, and $\bar{\eta}$ approaches infinity. Thus, Eq. (3) describes a relationship between changes of the exponent of the power law and the transition from solidlike $(x=1, \bar{\eta}=0)$ to fluidlike $(x=2, \bar{\eta}$ $=\infty$ ) behavior.

In the case of human airway smooth muscle (HASM) cells we converted the moduli $g^{\prime}$ and $g^{\prime \prime}$ (in units of $\mathrm{Pa} / \mathrm{nm}$ ) and viscosity $\mu$ (in Pa s/nm) into absolute moduli $G^{\prime}$ and $G^{\prime \prime}$ (in $\mathrm{Pa}$ ) and viscosity $M$ (in $\mathrm{Pa}$ s) using a geometric conversion factor, $\alpha$, as described above $[17,26]$. Thus, Eq. (3) can be reexpressed in the usual units of elastic moduli and viscosity with the transformations $G_{0}=\alpha g_{0}$, and $M=\alpha \mu$. This conversion does not impact the findings presented below, and has only been introduced so that our measurements can be compared with those of others. For other cell types we had not determined $\alpha$ and thus present our unconverted data ( $g^{\prime}$, $g^{\prime \prime}$, and $\mu$ ) only.

Cells. We measured the rheology of five different cell types: HASM cells, human bronchial epithelial (HBE) cells, mouse embryonic carcinoma cells (F9), mouse pulmonary macrophages (J774A.1), and human neutrophils. HASM cells were harvested from human trachea [27] and cultured as described in [28]. Confluent cells in passage 4-7 were serum-deprived for $24 \mathrm{~h}$. Cells were harvested with trypsin and plated at $37^{\circ} \mathrm{C}$ for 3 to $6 \mathrm{~h}$ in plastic wells $(6.4 \mathrm{~mm}$, 96-well Removawells, Immunlon, IL) at a density of 20000 cells/well. The wells were coated with collagen I (Cohesion, Palo Alto, CA) at a density of $500 \mathrm{ng} / \mathrm{cm}^{2}$. Approximately 10000 beads were added to an individual cell well $30 \mathrm{~min}$ prior to measurements of cell mechanics. Within one or two minutes, most beads sunk to the bottom of the well and were in contact with the apical surface of the cells. Before adding drugs or-if no drugs were added—before placing the wells into the microscope stage, the wells were washed twice with medium to remove unbound beads. Except where noted otherwise, the experimental protocol for all cell types was identical to that of HASM cells. HBE cells (Clonetics, San Diego, CA) isolated from the trachea and central airways were expanded to passage 4 in bronchial epithelial growth media (CC-3170, Clonetics). F9 cells (kindly supplied by Wolfgang Goldmann, Harvard Medical School, Boston, MA) were cultured as described in [29]. J774A.1 macrophages originated from a BALB/c/NIH mouse [30] and were obtained from the German Collection of Animal Cell Cultures (Tumorbank, DKFZ Heidelberg, Germany). J774A.1 cells were plated for $24 \mathrm{~h}$ in culture medium, followed by $24 \mathrm{~h}$ of bead incubation. Neutrophils were isolated from human blood obtained from consenting healthy adult volunteers by venipuncture into 0.1 vol of sodium citrate anticoagulant (Sigma), using Neutrophil Isolation Media (NIM; Cardinal Associates, Santa Fe, $\mathrm{NM}$ ). The resulting cell preparation contained $>95 \%$ neutrophils as assessed by hematoxylin and eosin staining. Neutrophils were suspended in Hanks Balanced Salt Solution and allowed to adhere on a glass surface for $10 \mathrm{~min}$, followed by $10 \mathrm{~min}$ of bead incubation. We performed all procedures with endotoxin-free solutions and completed all experiments within 3 hours of blood collection.

Protocol. The protocol for the twisting field application was as follows: We started with one cycle at $0.3 \mathrm{~Hz}$, followed by three cycles at $0.01 \mathrm{~Hz}$, five cycles each at 0.03 , $0.1,0.3$, and $0.75 \mathrm{~Hz}, 80$ cycles each at 4.2 and $10 \mathrm{~Hz}, 240$ cycles at $30 \mathrm{~Hz}, 800$ cycles at $100 \mathrm{~Hz}, 4800$ cycles at 300 $\mathrm{Hz}$, and 28800 cycles at $1000 \mathrm{~Hz}$. We then decreased the frequency, mirroring the protocol around $1000 \mathrm{~Hz}$. The last and the first $0.3 \mathrm{~Hz}$ cycle were not included in the data analysis. HBE cells, neutrophils, and macrophages were probed only at frequencies above $0.03 \mathrm{~Hz}$. Drugs were added 10 minutes prior to measurements of cell mechanics. Measurements were not repeated on the same wells under different drug treatment conditions.

Reagents. Tissue culture reagents and drugs used in this study were obtained from Sigma (St. Louis, MO, USA), with the exception of Trypsin-ETDA solution, which was purchased from Gibco (Grand Island, NY). Histamine, $\mathrm{N}$-formyl-methionyl-leucylphenylalanine (FMLP), and $\mathrm{N}^{6}, 2^{\prime}$-O-dibutyryladenosine $3^{\prime}, 5^{\prime}$-cyclic monophosphate (DBcAMP) were dissolved in distilled water at $0.1 M$. Cytochalasin $D$ was dissolved in DMSO at $0.1 M$. All reagents were frozen in aliquots, thawed on the day of use, and diluted in media.

Statistics. Equation (3) was fit to the data. In doing so, we had three objectives. The first was to estimate the parameters $g_{0}, \Phi_{0}, x$, and $\mu$. The second was to assess the extent to which changes in these parameters with defined interventions were statistically significant. The third objective was to establish the extent to which the number of free parameters could be reduced. In particular, stiffness data for different experimental conditions from cells of the same type seemed to indicate a value of $g_{0}$ and $\Phi_{0}$ that could be regarded as invariant with experimental interventions. Similarly, the friction data seemed to indicate that the viscosity parameter $\mu$ was nearly invariant with experimental interventions. As we 
show below, the number of free parameters could be reduced, although there was no reason a priori to think that this would be the case.

We used two complementary statistical analyses. The first and simplest used as a starting point pooled data in which at each frequency we considered only the median value of $\widetilde{g}$ across all cells for a given cell type and treatment condition. In the second approach, we used a mixed-effects analysis that deals explicitly with the measured frequency and drug response across all cells within a given cell type.

Pooled data analysis. We compared four models, each of which was based on Eq. (3). The four models differed only in regard to which parameters were held to be common among the conditions, and which were allowed to differ. In the first model all parameters $\left(x, g_{0}, \Phi_{0}\right.$, and $\left.\mu\right)$ were free in that there were no constraints across treatments. The second model constrained $g_{0}$ and $\Phi_{0}$ to be constant across treatments, while $x$ and $\mu$ were treatment-dependent. The third model further constrained the viscous coefficients $\mu$ to be equal among treatments and allowed only $x$ to vary. The fourth model further constrained $\Phi_{0}$ to be equal among all cell types, with $\Phi_{0}=2.14 \times 10^{7} \mathrm{rad} / \mathrm{s}$.

We observed that the variability in $\widetilde{g}$ between beads at a given frequency and treatment was approximately proportional to the magnitude of $\widetilde{g}$. This suggests using the logarithm of the complex stiffness measurements for data analysis and modeling. Furthermore, this approach was justified because the variability among cells has been shown to be distributed in a $\log$ normal fashion [14]. We used leastsquares minimization to evaluate the parameters in the fits. Explicitly, for each model we minimized the squared differences between the logarithm of the complex data and the logarithm of the complex model, summed over all frequencies and all treatment conditions; thus we minimized with regard to the sum of the logarithmic magnitude of $\widetilde{g}$ [the real part of $\log (\widetilde{g})]$ and the phase [the imaginary part of $\log (\widetilde{g})]$. We compared these models against one another by a reduction-in-variance F-test. Accordingly, the fractional reduction in the sum of the squared residuals $(S)$, suitably normalized by the appropriate degrees of freedom $\left(d_{f}\right)$, is an F-statistic, which we used to determine whether there was a statistically significant difference in the fits between the four models:

$$
F^{\mathrm{II}-\mathrm{I}}=\frac{\left(S^{\mathrm{II}}-S^{\mathrm{I}}\right) /\left(d_{f}^{\mathrm{II}}-d_{f}^{\mathrm{I}}\right)}{S^{\mathrm{I}} / d_{f}^{\mathrm{I}}},
$$

where the superscript I refers to the model with the greater number of parameters, and superscript II refers to the model with the reduced set of parameters. If the observed F-statistic exceeded a value corresponding to $p<0.05$, we concluded that the model with the greater number of parameters was significantly better than the other.

Mixed-effects model. To estimate confidence intervals of the parameters $x, g_{0}, \Phi_{0}$, and $\mu$, we performed a nonlinear mixed-effects analysis using the statistical analysis program $R$ (version 1.6.0). Equation (3) was fit to the logarithm of the complex modulus of the entire data set for a given cell type; this data set consisted of one $g^{\prime}$ and one $g^{\prime \prime}$ value for each
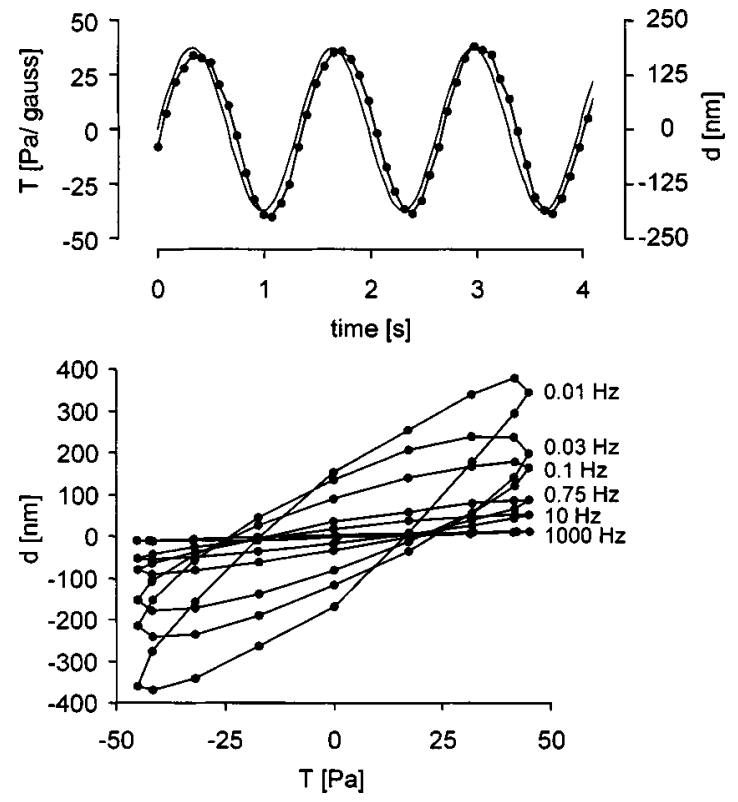

FIG. 2. (a) Specific torque $T$ (solid line) and lateral displacement $d$ (filled circles connected by a solid line) vs time in a representative bead. Bead displacement followed the sinusoidal torque with a small phase lag. The filled circles indicate when the image and data acquisition was triggered, which was 16 times per twisting cycle. (b) Loops of lateral bead displacement vs specific torque of a representative bead at different frequencies. With increasing frequency, displacement amplitude decreased.

bead at each frequency and treatment condition. A mixed effects model accounts for the random and systematic variation of $\widetilde{g}$ within a cell (at different frequencies) and between cells (systematic variability caused by drug treatment, and random, innate variability between cells) [31]. In particular, we allowed random variations of the parameter $g_{0}$ between beads.

\section{RESULTS}

\section{Bead movement}

Lateral bead movement lagged slightly behind the imposed torque [Fig. 2(a)]. When bead displacement was plotted versus torque, elliptical loops resulted [Fig. 2(b)]. Bead displacement amplitude in response to sinusoidal twisting varied widely between cells according to a log-normal distribution that has been analyzed in detail elsewhere [14], and resulted in a correspondingly wide distribution of $g^{\prime}$ and $g^{\prime \prime}$ between cells as described in more detail below. The phase angle between torque and bead displacement (or equivalently the hysteresivity $\eta$, which is the tangent of the phase angle), however, had a comparatively narrow distribution (see below).

When we increased twisting frequency while keeping torque amplitude constant, bead displacement amplitude decreased appreciably, whereas the phase angle changed relatively little [Fig. 2(b)].

\section{Linearity}

The relationship between torque amplitude and bead displacement amplitude was linear. When we varied the specific 


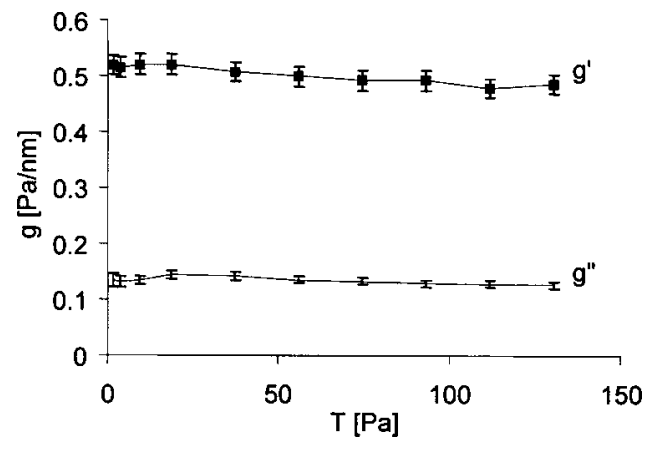

FIG. 3. $g^{\prime}$ and $g^{\prime \prime}$ vs specific torque $T$. $g^{\prime}$ and $g^{\prime \prime}$ were measured in 537 HASM cells at $f=0.75 \mathrm{~Hz}$. Specific torque amplitudes $T$ varied from 1.8 to $130 \mathrm{~Pa} . g^{\prime}$ and $g^{\prime \prime}$ were nearly constant, implying linear mechanical behavior of the cells in this range. Error bars indicate \pm one standard error.

torque amplitude between 1.8 and $130 \mathrm{~Pa}$ (at a twisting frequency of $0.75 \mathrm{~Hz}$ ), bead displacement amplitude changed in proportion, with the median amplitude increasing from 3 to $259 \mathrm{~nm}$; the phase lag between torque and bead displacement remained constant. Thus, over a wide range of specific torque amplitudes and bead displacement amplitudes, $g^{\prime}$ and $g^{\prime \prime}$ were constant, implying linear mechanical behavior of the cells in this range (Fig. 3). We observed similar behavior of $g^{\prime}$ and $g^{\prime \prime}$ at frequencies of $0.1 \mathrm{~Hz}$ and $10 \mathrm{~Hz}$ (data not shown). However, for bead displacement amplitudes in excess of $500 \mathrm{~nm}$, harmonics became evident, and both $g^{\prime}$ and $g^{\prime \prime}$ tended to increase, indicating the emergence of nonlinear behavior. We eliminated beads from subsequent analysis when the amplitude of higher harmonics exceeded $18 \%$ of the fundamental amplitude. Depending on the cell type, this was the case in about $2 \%$ to $20 \%$ of the beads. This approach is in keeping with that of Schmidt et al. and Choquet et al., who reported that fibronectin-coated microbeads could be sorted into two distinct populations: $80 \%$ of all beads appeared to be tightly bound to cytoskeletal structures, whereas $20 \%$ of the beads were loosely bound and could be easily moved in a laser trap $[32,33]$.

\section{HASM cells: Control conditions}

Figure 4 shows the relationship of $G^{\prime}$ and $G^{\prime \prime}$ versus frequency for HASM cells under control conditions. Each data point represents the median value of 256 cells; the variability between cells is addressed below. Throughout the frequency range studied $G^{\prime}$ increased with increasing frequency, $f$, according to a power law, $f^{x-1}$. Because the axes in Fig. 4 are logarithmic, a power-law dependency appears as a straight line with slope $x-1$. We argue below that if the CSK behaves as a soft glassy material, then $x$ would correspond to an effective noise temperature of the matrix.

The power-law exponent of $G^{\prime}$ was $0.20(x=1.20)$, indicating only a weak dependency of $G^{\prime}$ on frequency. $G^{\prime \prime}$ was smaller than $G^{\prime}$ at all frequencies except at $1 \mathrm{kHz}$. Like $G^{\prime}$, $G^{\prime \prime}$ also followed a weak power law with nearly the same exponent at low frequencies. At frequencies larger than 10 $\mathrm{Hz}$, however, $G^{\prime \prime}$ exhibited a progressively stronger frequency dependence, approaching but never quite attaining a

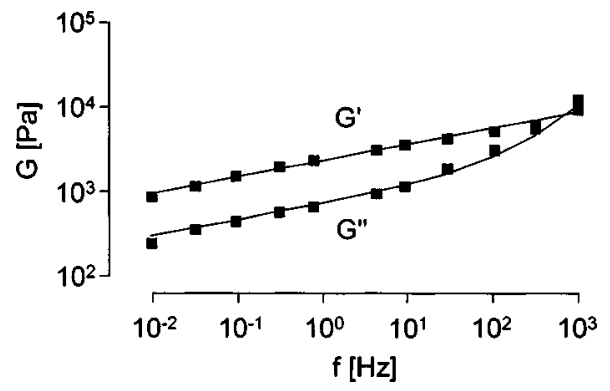

FIG. 4. $G^{\prime}$ and $G^{\prime \prime}$ (median of 256 HASM cells) under control conditions measured at frequencies between $0.01 \mathrm{~Hz}$ and $1000 \mathrm{~Hz}$. The solid lines were obtained by fitting Eq. (3) to the data. $G^{\prime}$ and $G^{\prime \prime}$ (in units of $\mathrm{Pa}$ ) were computed from the measured values of $g^{\prime}$ and $g^{\prime \prime}$ (in units of $\mathrm{Pa} / \mathrm{nm}$ ) times a geometric factor $\alpha$ of $6.8 \mu \mathrm{m}$ (see Methods). $G^{\prime}$ increased with increasing frequency, $f$, according to a power law, $f^{x-1}$, with $x=1.20 . G^{\prime \prime}$ was smaller than $G^{\prime}$ at all frequencies except at $1 \mathrm{kHz}$. At frequencies below $10 \mathrm{~Hz}, G^{\prime \prime}$ also followed a weak power law with nearly the same exponent as did $G^{\prime}$; above $10 \mathrm{~Hz}$ the power-law exponent increased and approached unity.

power-law exponent of 1 , which would be characteristic of a Newtonian viscosity.

\section{HASM cells: Challenge}

When cells were activated with the contractile agonist histamine $(100 \mu \mathrm{M}), G^{\prime}$ increased but still exhibited a weak power-law dependence on frequency; $x$ fell slightly to 1.17 (Fig. 5). When cells were relaxed with DBcAMP $(1 \mathrm{mM})$, $G^{\prime}$ decreased, and $x$ increased to 1.28 . When the actin cytoskeleton of the cells was disrupted with cytochalasin $D(2$

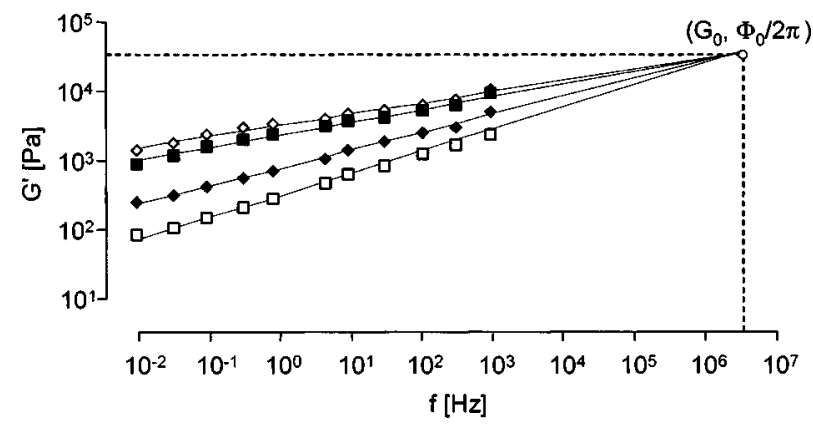

FIG. 5. $G^{\prime}$ vs $f$ in HASM cells under control conditions $(\boldsymbol{\square}, n$ $=256$ ), and after 10 min treatment with the contractile agonist histamine $\left(10^{-4} \mathrm{M}\right)(\diamond, n=195)$, the relaxing agonist DBcAMP $\left(10^{-3} \mathrm{M}\right)(\diamond, n=239)$ and the actin-disrupting drug cytochalasin $D\left(2 \times 10^{-6} \mathrm{M}\right)(\square, n=171)$. At all frequencies, treatment with histamine caused $G^{\prime}$ to increase, while treatment with DBcAMP and cytoD caused $G^{\prime}$ to decrease. Under all treatment conditions, $G^{\prime}$ increased with increasing frequency, $f$, according to a power law, $f^{x-1}$. $x$ varied between 1.17 (histamine) and 1.33 (cytoD). A decreasing $G^{\prime}$ was accompanied by an increasing $x$, and vice versa. Solid lines are the fit of Eq. (3) to the data. Surprisingly, these lines appeared to cross at a coordinate close to $\left[G_{0}, \Phi_{0} / 2 \pi\right]$, well above the experimental frequency range. According to Eq. (3), an approximate crossover implies that in the HASM cell the values of $G_{0}$ and $\Phi_{0}$ were invariant with differing treatment conditions. 


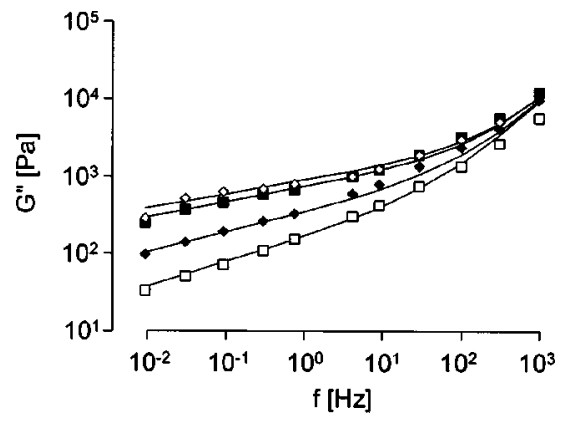

FIG. 6. $G^{\prime \prime}$ vs $f$ in HASM cells under control conditions ( $\square, n$ $=256)$, and after 10 min treatment with histamine $\left(10^{-4} \mathrm{M}\right)(\diamond$, $n=195), \operatorname{DBcAMP}\left(10^{-3} \mathrm{M}\right)(\diamond, n=239)$, and cytochalasin $D$ $\left(2 \times 10^{-6} \mathrm{M}\right)(\square, n=171)$. At all frequencies, treatment with histamine caused $G^{\prime \prime}$ to increase, while treatment with DBcAMP and cytoD caused $G^{\prime \prime}$ to decrease. Under all treatment conditions, $G^{\prime \prime}$ increased at frequencies below $10 \mathrm{~Hz}$ according to a power law, $f^{x-1}$, with exponents that were similar to that of the corresponding $G^{\prime}$ data (Fig. 5). Above $10 \mathrm{~Hz}$ the power-law exponents increased and approached unity for all treatments; the $G^{\prime \prime}$ curves merged onto a single relationship.

$\mu \mathrm{M}), G^{\prime}$ decreased further, and $x$ increased to 1.33 . Remarkably, the $G^{\prime}$ data defined a family of curves that, when extrapolated, appeared to intersect at a single value $\left(G_{0}\right)$ at a very high frequency $\left(\Phi_{0}\right)$ (Fig. 5).

With all drug treatments, $G^{\prime}$ and $G^{\prime \prime}$ tended to change in concert. The relationship between $G^{\prime \prime}$ and frequency remained a weak power law at lower frequencies, and the power-law exponent of $G^{\prime \prime}$ changed in concert with that of $G^{\prime}$. At the highest frequencies, the curves of $G^{\prime \prime}$ versus frequency for all treatments appeared to merge onto a single line with a power-law exponent approaching unity (Fig. 6).

\section{Structural damping equation: Reduction of variables}

When we fitted the structural damping equation to the median of the complex moduli of HASM cells, we obtained for each of the four treatment conditions a parameter set for $x, G_{0}, \Phi_{0}$, and $M$. The goodness of the fit was excellent $\left(r^{2}=0.997\right.$, Table I). Nonetheless, two observations indicated that the number of free parameters could be reduced without compromising the goodness of fit. First, we noticed that viscosity values $(M)$ were similar for all treatments (Table II, model 1), although cells after treatment with cy- tochalasin $D$ expressed a somewhat smaller viscosity. Second, the apparent common intersection of the $G^{\prime}$ versus $f$ curves at high frequencies (Fig. 5) suggested a single value $G_{0}, \Phi_{0}$ for all treatments. As explained below, this observation of a common intersection, or fixed point, turns out to have important consequences.

In order to test whether $G_{0}, \Phi_{0}$, and $M$ were indeed invariant with drug treatment, we analyzed the residual variances of the fit of Eq. (3) to the data. Specifically, we evaluated how well the structural damping equation [Eq. (3)] accounted for the pooled (median) data when either all parameters $\left(x, G_{0}, \Phi_{0}\right.$, and $\left.M\right)$ were free across drug treatment (model 1), when $G_{0}$ and $\Phi_{0}$ were constrained to be the same for all drug treatments (model 2), when $G_{0}, \Phi_{0}$, and $M$ were all constrained to be the same for all drug treatments (model 3), or when, in addition to the constraints of model 3, $\Phi_{0}$ was constrained to be the same for all cell types (model 4).

The residual variances of the fit of model 1 and model 2 to the pooled data were not significantly different from each other $(p=0.086$, Table I). Model 2 is thus to be preferred over model 1 because it can account for the data with fewer parameters. This finding supports the notion that $G_{0}$ and $\Phi_{0}$ were invariant with drug treatment. The residual variances $\left(r_{s s}\right)$ of the fit of model 3 and 4 to the data were slightly but significantly $(p<0.001)$ larger than those of models 1 or 2 , however (Table I). This indicates that the Newtonian viscosity $(M)$ term changed in response to drug treatment. These changes were small compared to drug-induced changes of $G^{\prime}$ and $G^{\prime \prime}$ at low frequencies, however. Moreover, as explained below, drug-induced changes of $\mu$ in cell types other than HASM did not reach statistical significance and, in the case of treatment with cytochalasin $D$, were inconsistent between cell types (Table III). Thus, for all practical purposes, $\mu$ could be regarded as being approximately invariant with drug treatment. Consequently, we were able to fit the structural damping equation to the entire data set depicted in Figs. 6 and 7 (solid lines) using only a single value of $G_{0}$, a single value of $\Phi_{0}$, and a single value of $M$, as shown in Table II. Despite this profound reduction in the number of parameters, the goodness of fit remained excellent $\left(r^{2}=0.993\right)$. Thus, all changes in $G^{\prime}$ and $G^{\prime \prime}$ that occurred with changes of frequency and in response to drug treatment were accounted for by changes of $x$ alone. A comparison between models 1-4 using a mixed-effect analysis of the bead-by-bead (nonpooled) data gave similar results (Table I, right). The goodness of fit $\left(r^{2}\right)$ of Eq. (3) to the data remained high for all

TABLE I. Statistical evaluation of the fit of the structural damping equation [Eq. (3)] to pooled (median) data and data from individual beads (mixed effects) in HASM cells. Models 1-4 denote different constraints of Eq. (3) (see text).

\begin{tabular}{|c|c|c|c|c|c|c|c|c|}
\hline & \multicolumn{4}{|c|}{ median } & \multicolumn{4}{|c|}{ mixed effects } \\
\hline & model 1 & model 2 & model 3 & model 4 & model 1 & model 2 & model 3 & model 4 \\
\hline$r^{2}$ & 0.997 & 0.997 & 0.993 & 0.993 & 0.948 & 0.948 & 0.947 & 0.947 \\
\hline $\mathrm{r}_{\mathrm{ss}}\left[\mathrm{Pa}^{2} / \mathrm{nm}^{2}\right]$ & 0.216 & 0.231 & 0.511 & 0.527 & 1028 & 1028 & 1047 & 1047 \\
\hline $\mathrm{df}$ & 76 & 78 & 81 & 82 & 18928 & 18930 & 18933 & 18934 \\
\hline $\mathrm{p}^{\mathrm{a}}$ & & 0.082 & $<0.0001$ & $<0.0001$ & & 0.001 & $<0.0001$ & $<0.0001$ \\
\hline
\end{tabular}

${ }^{\mathrm{a}}$ Compared to model 1 . 
TABLE II. Parameter estimates (mixed effects analysis) of the fit of the structural damping equation [Eq. (3)] to the data from individual beads on HASM cells. Models 1-4 denote different constraints of Eq. (3) (see text).

\begin{tabular}{lrrrr}
\hline \hline & model 1 & model 2 & model 3 & model 4 \\
\hline$G_{0}(\mathrm{kPa})$ & & 40.96 & 51.40 & 41.47 \\
$\Phi_{0}(\mathrm{rad} / \mathrm{s})$ & & $2.14 \times 10^{7}$ & $5.24 \times 10^{7}$ & $2.14 \times 10^{7}$ \\
$x$ control & 1.205 & 1.204 & 1.206 & 1.206 \\
$x$ hist & 1.165 & 1.166 & 1.171 & 1.170 \\
$x$ DBcAMP & 1.279 & 1.277 & 1.281 & 1.282 \\
$x$ cytoD & 1.327 & 1.329 & 1.319 & 1.320 \\
$M$ control (Pa s) & 0.68 & 0.68 & & \\
$M$ hist (Pa s) & 0.92 & 0.91 & & \\
$M$ DBcAMP (Pa s) & 0.73 & 0.74 & & \\
$M$ cytoD (Pa s) & 0.41 & 0.40 & & \\
$M$ common (Pa s) & \multicolumn{5}{c}{0.60} & 0.60 \\
\hline \hline
\end{tabular}

models ( $\left.r^{2}>=0.947\right)$, although model 1 gave a slightly better fit which, because of the very large number of data points $(n=18942)$, reached statistical significance $(p<0.001)$.

We estimated the confidence intervals of the fitted parameters from Eq. (3) using a nonlinear mixed-effect analysis (Table III). The $95 \%$ confidence interval for $G_{0}$ ranged from $4.91 \mathrm{~Pa} / \mathrm{nm}$ to $7.39 \mathrm{~Pa} / \mathrm{nm}$, and that of $\Phi_{0}$ ranged from $9.38 \times 10^{8} \mathrm{rad} / \mathrm{s}$ to $4.89 \times 10^{7} \mathrm{rad} / \mathrm{s}$ (Fig. 7). The $95 \%$ confidence interval for $x$ was $0.2 \%$ around the $x$ estimates, and the 95\% confidence interval for $M$ was $6 \%$ around the $M$ estimates. The parameter estimates obtained from the nonlinear mixed-effect analysis were in all cases close to the values obtained from the fit to the pooled data.

\section{Other cell types}

We measured the dependence of $g^{\prime}$ and $g^{\prime \prime}$ on frequency in four other cell types: F9 mouse embryonic carcinoma cells, human bronchial epithelial cells, J774A.1 mouse mac- rophages, and human neutrophils (Fig. 8). With the few exceptions noted below, these cell types behaved qualitatively much as did the HASM cells, although the numerical values of the fitted parameters differed (Table III). In all cell types, and regardless of intervention, $g^{\prime}$ increased according to a weak power law with exponent $x-1$, although $x$ differed among cell types.

Similarly, in all cell types $g^{\prime \prime}$ also followed a weak power law at low frequencies. As in the HASM cells, at the highest frequencies $g^{\prime \prime}$ approached a Newtonian-type viscous behavior, and the curves of $g^{\prime \prime}$ versus frequency from different treatments merged at the highest frequencies. Further, in all cell types and under all interventions, $g^{\prime}$ was larger than $g^{\prime \prime}$ for frequencies below $300 \mathrm{~Hz}$.

In all cell types, an increase in $g^{\prime}$ induced by drug treatment was accompanied by an increase of $g^{\prime \prime}$ and a decrease in $x$. Conversely, a decrease in $g^{\prime}$ was accompanied by a decrease of $g^{\prime \prime}$ and an increase of $x$. For example, when neutrophils were stimulated with FMLP $(10 \mathrm{nM}), g^{\prime}$ and $g^{\prime \prime}$ increased, and $x$ fell slightly. All cell types responded similarly to treatment with the actin-disrupting drug cytochalasin $D(2 \mu \mathrm{M}): g^{\prime}$ and $g^{\prime \prime}$ fell considerably, and $x$ increased.

Generally, the power-law exponent of $g^{\prime \prime}$ at low frequencies was similar to that of $g^{\prime}$, but we observed some systematic departures from that behavior. In three cell types (human bronchial epithelial cells, macrophages, and neutrophils), we found that the behavior of $g^{\prime \prime}$ under baseline conditions at frequencies below $10 \mathrm{~Hz}$ departed systematically from the power-law behavior implied by Eq. (3); in those cell types $g^{\prime \prime}$ became nearly frequency-independent.

In all cell types, the $g^{\prime}$ versus $f$ curves for different drug treatments intersected at a very high frequency $\left(\Phi_{0}\right)$. The estimated values for $\Phi_{0}$ differed between cell types, but the 95\% confidence intervals of $\Phi_{0}$ were wide and overlapped (Fig. 7). These wide confidence intervals arose because the range of power-law slopes was small. Accordingly, the structural damping equation [Eq. (3)] was able to fit the data in all

TABLE III. Parameter estimates and 95\% confidence intervals from a mixed effects analysis of the fit of the structural damping equation (Eq. 3, constraint model 2) to the data from individual beads on different cell types.

\begin{tabular}{|c|c|c|c|c|c|}
\hline & F9 & HASM & HBE & Macro & Neutro \\
\hline$g_{0}(\mathrm{~Pa} / \mathrm{nm})$ & $0.52_{0.26}^{1.04}$ & $6.02_{4.91}^{7.39}$ & $2.86_{2.08}^{3.93}$ & $2.61_{1.30}^{5.25}$ & $2.50_{1.14}^{5.48}$ \\
\hline$\phi_{0}(\mathrm{rad} / \mathrm{s})$ & $3.20 \times 10^{6} \begin{array}{l}5.70 * 10^{8} \\
1.80 * 10^{4}\end{array}$ & $2.14 \times 10^{7} \begin{array}{l}4.89 * 10^{7} \\
9.38 * 10^{6}\end{array}$ & $1.09 \times 10^{6} \begin{array}{l}3.92 * 10^{5} \\
3.00 * 10^{5}\end{array}$ & $6.86 \times 10^{4} \begin{array}{l}8.60 * 10^{5} \\
5.47 * 10^{3}\end{array}$ & $1.90 \times 10^{7} \begin{array}{l}7.05 * 10^{8} \\
5.14 * 10^{5}\end{array}$ \\
\hline $\begin{array}{l}x \text { control } \\
x \text { FMLP }\end{array}$ & $1.195_{1.190}^{1.200}$ & $1.204_{1.202}^{1.206}$ & $1.173_{1.169}^{1.178}$ & $1.200_{1.191}^{1.209}$ & $\begin{array}{l}1.186_{1.178}^{1.194} \\
1.157_{1.148}^{1.166}\end{array}$ \\
\hline $\begin{array}{l}x \text { histamine } \\
x \text { DBcAMP }\end{array}$ & & $\begin{array}{l}1.166_{1.163}^{1.189} \\
1.277_{1.275}^{1.280}\end{array}$ & & & \\
\hline$x$ cytoD & $1.302_{1.294}^{1.310}$ & $\begin{array}{l}1.329_{1.326}^{1.1332} \\
\end{array}$ & $1.319_{1.315}^{1.323}$ & $1.338_{1.327}^{1.348}$ & $1.252_{1.246}^{1.259}$ \\
\hline$\mu$ control $^{\mathrm{a}}$ & $0.47_{0.43}^{0.51}$ & $1.00_{0.94}^{1.07}$ & $0.64_{0.59}^{0.69}$ & $1.95_{1.71}^{2.22}$ & $0.63_{0.56}^{0.71}$ \\
\hline$\mu$ FMLP $^{\mathrm{a}}$ & & & & & $0.82_{0.73}^{0.93}$ \\
\hline$\mu$ histamine $^{\mathrm{a}}$ & & $1.34_{1.25}^{1.44}$ & & & \\
\hline$\mu \mathrm{DBcAMP}$ & & $1.09_{1.04}^{1.14}$ & & & \\
\hline$\mu$ cytoD $^{\mathrm{a}}$ & $0.65_{0.60}^{0.70}$ & $0.59_{0.56}^{0.62}$ & $0.55_{0.51}^{0.59}$ & $3.02_{2.71}^{3.35}$ & $0.86_{0.92}^{0.81}$ \\
\hline
\end{tabular}

${ }^{\mathrm{a}}$ In $10^{-4} \mathrm{~Pa} \mathrm{~s} / \mathrm{nm}$. 


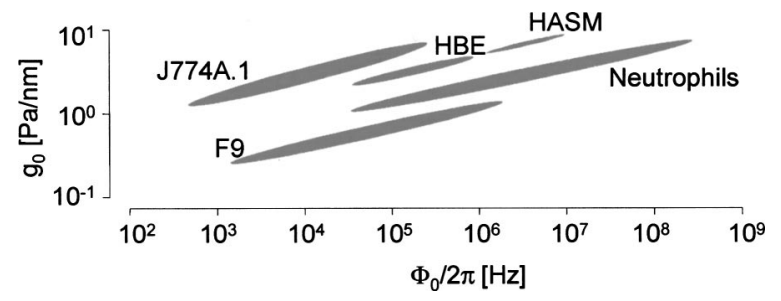

FIG. 7. $95 \%$ confidence region ellipses for $\Phi_{0}$ (in $\mathrm{Hz}$ ) and $g_{0}$ in five different cell types as estimated with a mixed-effects analysis. The confidence intervals for $\Phi_{0}$ were very wide and overlapped between different cell types.

cell types using only a single value for $\Phi_{0} \quad(2.14$ $\times 10^{7} \mathrm{rad} / \mathrm{s}$ ) without appreciably decreasing the goodness of fit (Table I, model 4). The stiffness $g_{0}$ differed between cell types, as did the high-frequency viscosity $\mu$ (Table III). Among all cell types, macrophages turned out to have the highest $\mu$ value, whereas mouse embryonic carcinoma cells (F9) had the lowest $g_{0}$ value and $\mu$ value. As in HASM cells, $\mu$ changed only little with drug treatment (Table III). In all cell types, therefore, the relationships of $g^{\prime}$ and $g^{\prime \prime}$ versus frequency and their changes with drug treatments were well fit by Eq. (3) (model 4) using only one free parameter, $x$ (Fig. 8 solid lines).

\section{Master curves: Scaling the data}

In order to compare responses between drug interventions and between differing cell types, we normalized the data as follows. We defined a normalized cell stiffness $G_{n}$ as $g^{\prime}$ measured at $0.75 \mathrm{~Hz}$ (an arbitrary choice) divided by $g_{0}$, where $g_{0}$ served as an internal stiffness scale for each cell type; this normalization causes the geometric factor $\alpha$ to cancel out. To normalize the cell's frictional properties, we used the ratio $g^{\prime \prime} / g^{\prime}$ (the hysteresivity $\eta$ ) at $0.75 \mathrm{~Hz}$, which also causes the geometric factor $\alpha$ to cancel out. We estimated $x$ from the fit of Eq. (3), model 1 (no constraints), to the pooled (median) data. We then plotted $G_{n}$ versus $x$ and $\eta$ versus $x$ (Fig. 9, black symbols).
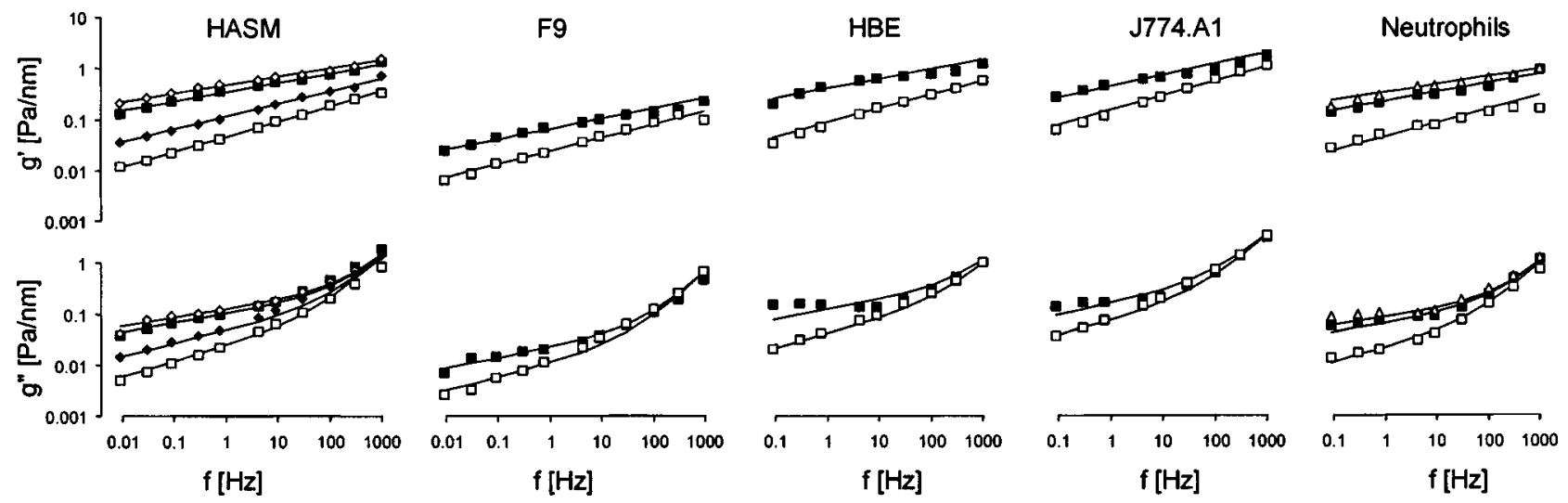

FIG. 8. $g^{\prime}$ and $g^{\prime \prime}$ vs frequency in five different cell types under control conditions $(\mathbf{\square})$, and after 10 min treatment with histamine $\left(10^{-4} \mathrm{M}\right)(\diamond)$, DBcAMP $\left(10^{-3} \mathrm{M}\right)(\diamond)$, cytochalasin $D\left(2 \times 10^{-6} \mathrm{M}\right)(\square)$, and FMLP $\left(10^{-8} \mathrm{M}\right)(\triangle)$. The solid lines are the fit of $x$, $\mu$, and $g_{0}$ [according to Eq. (3), constraint model 4] to the data. In all cells, $\Phi_{0}$ was set to $2.14 \times 10^{7} \mathrm{rad} / \mathrm{s}$, and $\mu$ was set to be constant within a given cell type (i.e., independent of drug treatment). In all cell types, the relationships of $g^{\prime}$ and $g^{\prime \prime}$ vs frequency and their changes with drug treatments were well fit by Eq. (3) using only one free parameter, $x$. However, small but systematic discrepancies between the $g^{\prime \prime}$ data and the fit are noted in HBE cells, J741.A cells, and neutrophils (see text). 

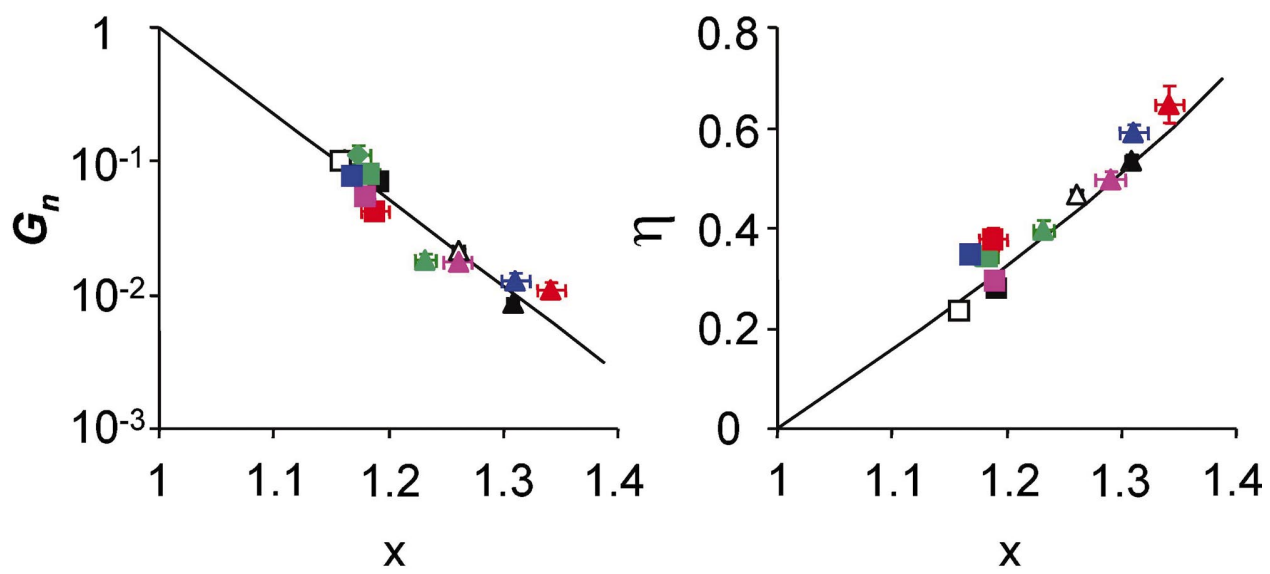

FIG. 9. (Color) Normalized stiffness $G_{n}$ (left) and hysteresivity $\eta$ (right) vs $x$ of HASM cells (black, $n=256$ ), human bronchial epithelial cells (blue, $n=142$ ), mouse embryonic carcinoma cells (F9) cells (pink, $n=50$ ), mouse macrophages (J774A.1) (red, $n=46$ ), and human neutrophils (green, $n=42$ ) under control conditions $(\boldsymbol{\square})$, treatment with histamine $(\square)$, FMLP $(\diamond)$, DBcAMP $(\triangle)$, and cytochalasin $D(\mathbf{\Delta})$. $x$ was obtained from the fit of Eq. (3), model 1 (no constraints), to the pooled (median) data. Drugs that increased $x$ caused the normalized stiffness $G_{n}$ to decrease and hysteresivity $\eta$ to increase, and vice versa. The normalized data for all types collapsed onto the same relationships. The structural damping equation by Eq. (3) is depicted by the black solid curves: $\ln G_{n}=(x-1) \ln \left(\omega / \Phi_{0}\right)$ with $\Phi_{0}=2.14$ $\times 10^{7} \mathrm{rad} / \mathrm{s}$, and $\bar{\eta}=\tan ((x-1) \pi / 2)$. Error bars indicate \pm one standard error. If $x$ is taken to be the noise temperature, then these data suggest that the living cell exists close to a glass transition and modulates its mechanical properties by moving between glassy states that are "hot," melted, and liquidlike, and states that are "cold," frozen, and solidlike. In the limit that $x$ approaches 1 the system behaves as an ideal Hookean elastic solid, and in the limit that $x$ approaches 2 the system behaves as an ideal Newtonian fluid [Eq. (3)].

Surprisingly, the value of $x$ derived from any given bead under a given treatment condition covaried with the value of $g^{\prime}$ (Fig. 10). Cells with smaller $g^{\prime}$ tended to have lower $x$ values, and cells with larger $g^{\prime}$ tended to have higher $x$ values. This behavior was different from the behavior seen in population averages, where an increase of $x$ in response to drug challenge was consistently associated with a decrease in $g^{\prime}$ (Fig. 10; population averages depicted by large symbols).

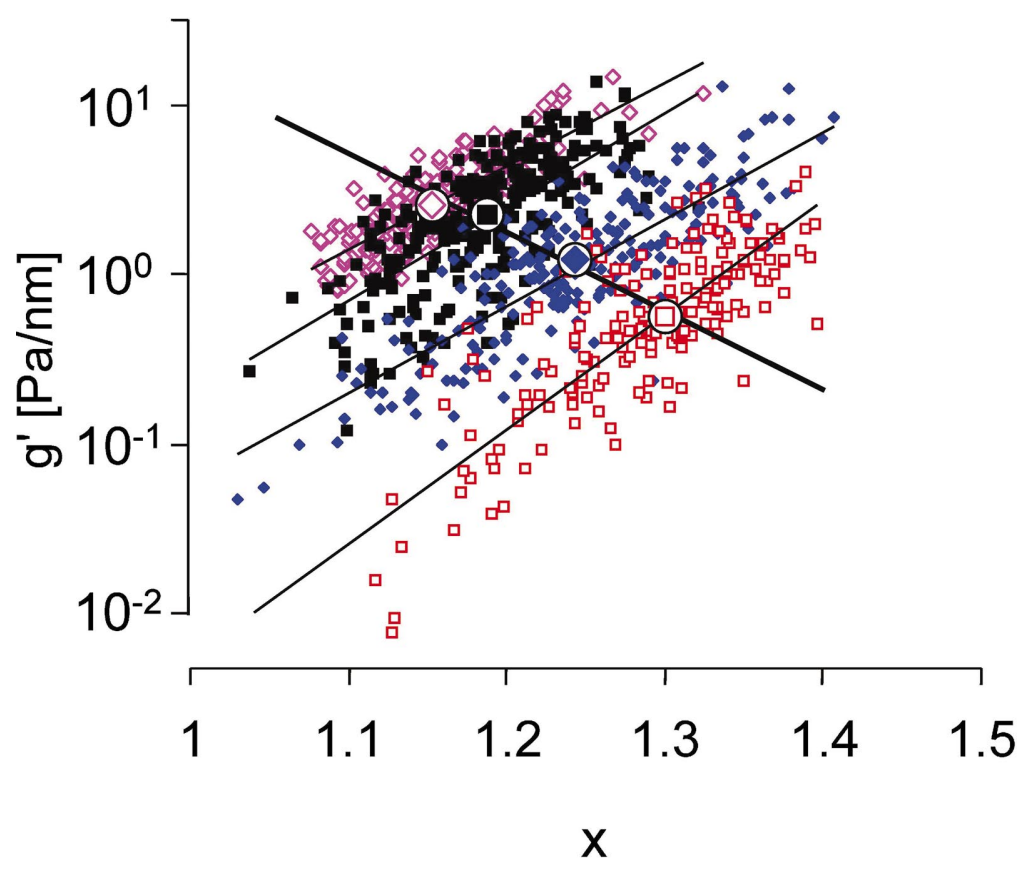

\section{DISCUSSION}

We have measured the elastic $\left(g^{\prime}\right)$ and the frictional $\left(g^{\prime \prime}\right)$ moduli of five different cell types over five frequency decades and with a variety of interventions. Our principal findings are as follows. Both $g^{\prime}$ and $g^{\prime \prime}$ increased with probing frequency according to a weak power law. This frequency dependence was well described by the structural damping equation [Eq. (3)]. Data from all cells, all frequencies, and all experimental conditions could be scaled in such a way that
FIG. 10. (Color) Relationship between $g^{\prime}$ vs $x$ in HASM cells under control (ם) conditions, and after treatment with histamine $(\diamond)$, DBcAMP $(\diamond)$, and cytochalasin $D(\square) . g^{\prime}$ of individual beads were measured at $1 \mathrm{kHz}$. $x$ was determined from the exponent of a power-law regression to the $g^{\prime}$ data of individual beads measured between $0.01 \mathrm{~Hz}$ and $1 \mathrm{kHz}$. Each small symbol represents the data from one bead, while the large symbols represent the median of all beads measured under one condition. The thin solid lines are the exponential regression through the data for individual beads. The correlation coefficient $\left(r^{2}\right)$ for the regression was 0.48 under control conditions, and 0.68 under all other conditions. The correlation coefficients decreased when the relationships were analyzed for $g^{\prime}$ values measured at lower frequencies than $1 \mathrm{kHz}$, but remained significant for $g^{\prime}$ values measured above $0.3 \mathrm{~Hz}$ ( $p$ $<0.05)$. The thick solid line is the exponential regression through the median values. 


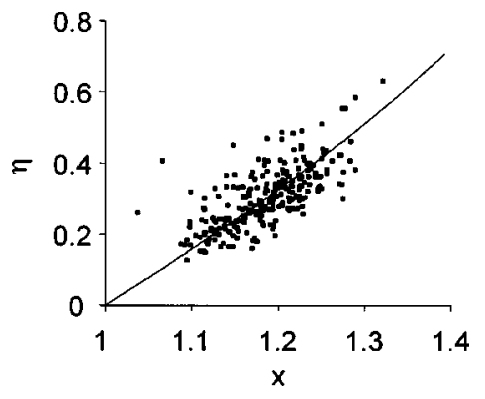

FIG. 11. Relationship between hysteresivity $\eta$ vs $x$ in HASM cells under control conditions. Each symbol represents the data from one bead $(n=256)$. $\eta$ was determined from the ratio $g^{\prime \prime} / g^{\prime}$ of individual beads measured at $0.75 \mathrm{~Hz}$. $x$ was determined from the exponent of a power-law regression to the $g^{\prime}$ data of individual beads measured between $0.01 \mathrm{~Hz}$ and $1 \mathrm{kHz}$. The solid line represents the relationship between $\eta$ and $x$ as predicted by the structural damping equation [Eq. (3)]: $\eta=\tan (\pi / 2(x-1))$.

they collapsed onto two master relationships, one describing stiffness and the other friction; to a reasonable approximation, changes of stiffness and friction induced by pharmacological interventions were accounted for solely by changes in only one parameter, namely the power-law exponent $x$. The rheology of living cells in culture was seen to differ in a qualitative fashion from the rheology that has been reported for reconstituted actin gels containing specific actin crosslinkers. These latter simplified systems have played an important role in developing our understanding of the functions of specific proteins $[34,35]$ but at the same time, they fail to capture some essential aspects of the biology.

In this section, we critique the methods that were employed and contrast our findings against those reported in the literature. We then go on to discuss time-scale invariance and the collapse of all data onto master curves, wherein the measured exponent $x$ is shown to play a major unifying role leading in turn to a major empirical simplification. These are experimental findings, they are model-independent, and they stand independently of interpretation or putative mechanism.

With regards to mechanism, we conclude by showing that the mechanical behavior reported here satisfies many of the empirical criteria that define the class of soft glassy materials [7]. If the CSK is indeed a soft glass, then the theory of soft glassy rheology (SGR) would identify the parameter $x$ as being an effective temperature of the matrix, distinct from the thermodynamic temperature. Moreover, SGR theory leads to a novel mechanistic perspective on the nature of protein-protein interactions and their contribution to integrated mechanical functions of the intact living cell, including the ability of the cytoskeleton to deform, flow, and remodel.

\section{Critique of methods}

The data presented here were not entirely general. Our studies were restricted to only five types of adherent cells and to small sinusoidal stresses and deformations. As a probe we used beads of only a single size $(4.5 \mu \mathrm{m})$ that were coupled to the cytoskeleton via integrins. The binding be- tween integrin receptors and ligands on the bead may have induced changes of cell mechanics. Further, these studies were limited in that we did not discriminate mechanical responses based on cell size, shape, or location of the bead on the cell, all of which have been shown to influence cell mechanics $[11,32,38,37]$. It is possible, therefore, that our results might be not so much a reflection of intrinsic properties of the cytoskeleton but instead are peculiar to the probe that we used, or are attributable to receptor kinetics or membrane mechanics. The evidence described below argues against those possibilities, however.

Binding of RGD-coated beads with integrin receptors on the cell surface has been shown to trigger a host of cellular response that are likely to have time-dependent effects on cell mechanics, including but not limited to focal adhesion formation, sodium-proton antiporter activation, increase in cyclic adenosine monophophate levels, protein kinase A translocation, and a multitude of gene activation [11,32,3840]. These responses can be considered as entirely physiologic, however, in that they closely mimic the responses observed when these cells adhere to and spread on dishes coated with integrin ligands [38]. To separate mechanical responses that may have been triggered by ligand binding events from measurements of passive cell mechanics, we employed four strategies. First, we investigated the effects of different bead-coatings, including extracellular matrix proteins (vitronectin and collagen), activating antibodies, and, importantly, nonactivating antibodies to different integrin subunits (data not shown). Despite the differences in the signaling cascades that may be activated by these different ligands, in each case power-law behavior prevailed (M. Puig de Morales, personal communication). Therefore, the existence of power-law behavior did not depend on the details of the coupling of the bead to the cell. Second, we carefully controlled the timing so that measurements were performed 30 minutes after bead addition ( $24 \mathrm{~h}$ in the case of macrophages). Third, synchronous detection of bead movement was used to distinguish periodic bead motions caused by the magnetic field from any nonsynchronous bead movements, guaranteeing that we measured purely mechanical responses to the mechanical perturbations of the cells. Finally, each measurement on each bead was performed at two time points so that the effects of twist versus the effects of time could be discerned.

We found that cell rheology greatly changed after treatment with drugs that altered actin polymerization or actomyosin bridge formation. In particular, we found that in response to the contractile agonist histamine smooth muscle cells increased stiffness by 1.4-fold, in response to DBcAMP decreased stiffness by 3.4-fold, thus confirming that these cells express a considerable degree of contractile tone at baseline, and in response to cytochalasin $D$ decreased stiffness by 8.3 -fold (at $0.75 \mathrm{~Hz}$ ), Other cell types responded to cytochalasin $D$ with a decrease in stiffness of at least threefold. Neutrophils increased stiffness in response to FMLP by 1.4-fold. In all cases cell stiffness changed in the direction one would expect based on the well-characterized effects of these drugs on the cytoskeleton. Moreover, these results are in good agreement with a large number of studies that report 
the effect of these drugs on cell mechanics but employed different measurement techniques, including indentation with a glass fiber [41], scanning atomic force microscopy [42], and measurement of the transit time of cells through capillary pores [43]. In a separate set of experiments we also used beads coated with acetylated low-density lipoprotein (acLDL); those beads bind to scavenger receptors which do not form focal adhesion complexes $[11,40]$. We found that baseline values of $G^{\prime}$ and $G^{\prime \prime}$ measured with acLDL-coated beads were smaller by fivefold compared with beads bound to integrins, and demonstrated an attenuated response to contractile agonists (data not shown).

Taken together, these findings support the conclusion that cell stiffness and friction as reported here predominantly reflect the mechanical properties of cytoskeletal structures. These data cannot be explained by receptor-ligand dynamics as would be the case if there were repetitive peeling of the bead away from the cell surface and subsequent reattachment with each bead oscillation. Neither can these data be readily explained by changes in cell membrane mechanics. Rather, these data are more consistent with the notion that the bead binds avidly to the cytoskeleton via focal adhesions, and that the cytoskeleton is deformed as the bead rotates (Fig. 1). This view is in accord with those of Schmidt et al. [32] and Plopper et al. [40], who showed that beads bound to the apical cell surface via $\beta 1$ integrins are tightly connected to deep cytoskeletal structures via a series of protein intermediates. Moreover, several studies have demonstrated that the stiffness of smooth muscle cells probed with magnetic beads bound to $\beta 1$ integrins can be modulated over a wide range by inhibiting or activating myosin motors $[13,14,28,44-47]$.

Variability. Values for stiffness $\left(g^{\prime}\right)$ and friction $\left(g^{\prime \prime}\right)$ between cells of the same type and under the same treatment varied over more than two orders of magnitude (geometric $\mathrm{SD}=2.1$, Fig. 10). This variability may have been caused by differences in the attachment geometry as reflected by the scaling factor $\alpha$, local variations of cell mechanics, proximity of neighboring cells, variations in bead size or magnetic moment. Nonetheless, we found that the behavior in individual cells of every cell type and under all treatment conditions conformed well to the structural damping equation [Eq. (3)], regardless of the magnitude of $\widetilde{g}$ (Tables I-III). The variability of $x$ between cells for a given drug treatment was small $(\mathrm{SD}<0.05)$.

The value of $x$ derived from any given bead under a given treatment covaried with the magnitude of the measured stiffness, however (Fig. 10). Beads reflecting higher stiffness tended to exhibit stronger frequency dependence (corresponding to higher values of $x$ ) compared to those reflecting smaller stiffness. We speculate that greater stiffness may be indicative of a deeper internalization of the bead into the cell body and a closer association of the bead with the internal cytoskeleton, while smaller stiffness may be indicative of little internalization of the bead and its association mainly with cortical structures $[14,17,26]$. If true, then the covariance between $x$ and $g^{\prime}$ may be attributable to differences between cortical and internal cytoskeletal structures, whereby the cortical cytoskeleton (as probed by marginally internalized beads with smaller $g^{\prime}$ ) exhibits smaller $x$ values, and the internal cytoskeleton (as probed by deeply internalized beads with larger $g^{\prime}$ ) exhibits larger $x$ values. Below we argue that $x$ may reflect the degree of metastability of the cytoskeletal matrix. If true, then these data would suggest that the cortical cytoskeleton is more stable than is the internal cytoskeleton.

Linearity. We found that loops of bead displacement versus specific torque appeared as simple ellipses in most cells [Fig. 2(b)], and that $g^{\prime}$ and $g^{\prime \prime}$ did not change appreciably as specific torque amplitude was varied between 1.8 and $130 \mathrm{~Pa}$ (Fig. 3). Therefore, cell mechanics could be regarded as being linear over the range of stresses and deformations employed in this study. We did find departures from linear behavior in some beads with displacement amplitudes in excess of $500 \mathrm{~nm}$, however. These beads caused larger cellular deformations around their attachment site, and they rotated more than beads with smaller displacement amplitudes [17]. A nonlinear torque-displacement relationship in those beads could arise from three sources: geometric nonlinearities induced at large deformations, intrinsic nonlinearities of the material, and the dependence of torque on the cosine of the angle between bead magnetization and the direction of the twisting field [Eq. (1)] [17,26]. In this study we excluded all beads with large displacement amplitude from further analysis. For the remaining beads, which was more than $80 \%$ of the total, we found linear responses (Fig. 3) and thus can rule out nonlinearities of the cell or of the measurement technique.

The finding of linear or nearly linear cell mechanics is consistent with reports by Bausch et al., who probed macrophages over forces ranging from $500 \mathrm{pN}$ to $2 \mathrm{nN}$ using magnetic tweezers [48], by Thoumine and Ott, who imposed strains of over 1.5 onto fibroblasts that were held between force plates [49], and by Alcaraz et al., who probed endothelial cells with atomic force microscopy over indentation forces ranging from $0.1 \mathrm{nN}$ to $0.9 \mathrm{nN}$ [50].

It is interesting, however, that the finding of linear mechanical behavior would appear to be in conflict with the view that cells express inherently nonlinear mechanical behavior, including shear thinning and strain hardening [51,52]. Although this view is widely held, evidence in the literature demonstrating nonlinearities is scant and can be traced to two primary sources. First, strain hardening is occasionally confused with the increase in stiffness that is seen in many cell types after they are mechanically stimulated. The increase in stiffness is the result of active responses of the cell to these stimuli, such as strengthening of the focal adhesion sites after beads attached to integrins were immobilized in optical traps [33], cytoskeletal remodeling after exposure to fluid shear flow [53], or contractile activation of cells that are compressed or stretched between microplates [49]. These active responses of the cell do not become evident until several seconds to hours after the onset of the mechanical stimulus, and are distinct from the passive mechanical responses of the cytoskeleton we have measured here. Second, reports from our own laboratory are often cited to support strain hardening $[11,12,54,55]$. We have recently shown, however, that this apparent strain-hardening is an artifact that comes about when an external magnetometer is used to measure the aver- 
age rotation of the entire bead population within a cell well containing several thousands of beads and a like number of cells. That population inevitably includes a small percentage of beads that are poorly bound to the cells $[14,26,56] \mathrm{Be}-$ cause they are loosely bound, these beads can rotate extensively even at very low twisting fields, and contribute substantially to the net magnetic signal. But because they cannot rotate in excess of 90 degrees, their contribution quickly saturates at a higher twisting field. The mistaken impression of strain hardening arises from an increasing number of loosely bound beads that saturate as the twisting field is increased. Here, by contrast, we have presented evidence that individual cells, when probed with firmly attached beads, display linear material properties over a wide range of specific torque amplitudes between 1.8 and $130 \mathrm{~Pa}$. While this result does not rule out nonlinear material behavior at higher stresses or strain rates, we conclude that the apparent strainhardening previously reported with magnetic twisting cytometry is an artifact arising from population averages that include loosely bound beads $[13,26]$. This artifact is avoided by using optical detection of bead motion, which allows us to exclude beads from further analysis when they are poorly bound to the cell, or when they are arranged in clusters or in close proximity to neighboring beads.

Compatibility with previous reports. The power-law dependence of $g^{\prime}$ and $g^{\prime \prime}$ on frequency reported here is consistent with data reported for atrial myocytes and fibroblasts measured with atomic force microscopy (AFM), for pellets of mouse embryonic carcinoma cells measured with a disk rheometer, for airway smooth muscle cells measured with oscillatory magneto-cytometry, and for kidney epithelial cells measured by laser tracking of Brownian motion of intracellular granules [13,36,57-59]. Yamada et al. also measured cell mechanics before and after microfilaments were disrupted with Latrunculin A, and obtained two curves of $g^{\prime}$ versus $f$ that intersected at a frequency comparable to the value we report here [36].

Of particular relevance are data recently reported for human bronchial endothelial cells measured over frequencies ranging from $0.01 \mathrm{~Hz}$ to $100 \mathrm{~Hz}$ with atomic force microscopy [50]. The AFM probe used in that study was uncoated and, unlike previous reports using AFM, the measurements were corrected for the viscous forces of the cell culture medium acting on the AFM cantilever. These data conformed to the structural damping equation [Eq. (3)] and were entirely consistent with the data reported here. Moreover, friction in those cells approached the behavior of a viscous fluid at a frequency range comparable to our findings.

Taken together, data reported previously in different cell types, measured with techniques that are fundamentally different in regard to probe size, coupling to the cell, and amplitude of deformations, strongly suggest that the results reported here are not peculiar to the measurement methodology and instead reflect intrinsic cytoskeletal properties. Compared with other methods that are available to probe cell mechanics, the method used here has the particular advantage to be able to measure responses of large numbers of cells in a short time and over a wide frequency range. However, because the position at which the beads settle onto the cell surface cannot be controlled, our method offers only a limited ability to resolve local variations in cell mechanical responses.

\section{Time-scale invariance}

With the few exceptions highlighted below, in all cases reported here cells conformed to the structural damping equation [Eq. (3)]. When $g^{\prime}$ and $g^{\prime \prime}$ were measured over a wide range of frequencies, in the resulting data no special frequency, or molecular relaxation time, or resonant frequency stood out. Instead, $g^{\prime}$ and $g^{\prime \prime}$ increased with frequency in a featureless fashion following a weak power law, $f^{x-1}$. Except for a small additive viscous term that emerged only at high frequencies, mechanical responses were not tied to any specific frequency scale, and in that respect can be regarded as being scale-free. Scale-free dynamics stands in contrast with the frequency-dependent properties of common polymer matrices, whose spectra typically display distinct plateaus, shoulders and inflections; these features are indicative of transitions from one dominant molecular mechanism to another [60]. For example, rubbers show a transition from dominance of chain entropy at lower frequencies, where stiffness is in the MPa range, to dominance of van der Waals bonds at higher frequencies, where stiffness is in the GPa range. Such transitions do not occur in scale-free media, in which case molecular relaxation processes in the matrix are not tied to any particular internal time scale or any distinct molecular rate process.

In that connection, the inverse Fourier transform of the structural damping equation predicts that stress relaxation after a unit step deformation should follow a power law in time, $t^{1-x}$ [Eq. (2)], and that displacements in response to a unit step torque (i.e., the creep response) follow a similar power law, $t^{x-1}$. Experiments in which we have measured bead displacements in response to a step change of bead torque have confirmed power-law creep responses in HASM cells over more than three decades of time $(3 \mathrm{~ms}$ to 3.2 seconds; G. Lenormand, personal communication). Powerlaw stress relaxation, power-law creep, and structural damping behavior have been reported in a variety of inert and biological materials [20-22,25,61-64].

Most studies of single cell rheology report data that span only relatively small ranges of time or frequency, typically one or at most two decades. Almost invariably, authors have fit simple viscoelastic models embodying a small number of distinct elastic and viscous components to these data and necessarily, these models express only a limited range of characteristic relaxation times [11,12,37,48,54,55,65-69]. Associated model parameters were often given physical interpretations such as cytoplasmic viscosity $[10,37,49,69-74]$, static elasticity of the cytoskeleton $[11,12,49,54,55,75]$, or tension, surface elasticity and bending modulus of the cell cortex $[71,72,74,76]$. Reported values for the model parameters, however, varied greatly between different visco-elastic models, experimental methods and conditions, and cell types studied [52,67,68,70,73].

Scale-free rheological behavior as reported here calls into question all such models and associated interpretations. 
When power-law behavior prevails, the fit of any lumped linear visco-elastic model to data obtained over a limited range of frequency (or time) will result in apparent relaxation time scales that are determined mostly by the time (or frequency) range probed in the experiment, as opposed to the material properties themselves [77]. That is to say, whatever limited range might be probed, one or two time constants in that range will always be found to fit the available data. Equivalently, with data spanning only a limited time or frequency range, it is difficult to distinguish an exponential law from a power law. Not surprisingly therefore, those reports in which cell responses have been measured over a large range of frequency or time have systematically abstained from using simple visco-elastic models to fit their data $[36,50,57-$ 59].

It has been suggested by Buxbaum et al. and Tsai et al. that the cytoplasm does not behave as a Newtonian fluid but exhibits power-law shear thinning, and that measurements of cell rheology do not so much depend on the frequency or time scale of the imposed forces or deformations but instead depend on the shear rate $[52,73]$. To test this possibility, we probed cells over a large range of shear rates using two different protocols; first we changed the deformation amplitude while maintaining a constant frequency, and second we changed the frequency while maintaining a constant amplitude. We found no shear thinning when the deformation amplitude was changed and the probing frequency was kept constant (Fig. 3). However, cell rheology did vary according to a power law with frequency when the deformation amplitude was kept constant (Fig. 4). Thus, our measurements of cell rheology did not depend on shear rate but instead on the time scale or frequency range over which the shear was applied.

\section{Other invariants of cell mechanical behavior: Collapse onto master curves}

Our measurements spanned five frequency decades, five different cell types and challenges with a variety of drugs. Nonetheless, the mechanical behavior of cells was restricted to vary only in a very particular way [Eq. (3)], and with only a small set of options to explain differences in mechanical properties: the power-law exponent $x$, the viscosity $\mu$, and scale factors for stiffness $\left(g_{0}\right)$ and frequency $\left(\Phi_{0}\right)$. A major finding of this study is that from those options, the parameter $x$ alone was to a reasonable extent sufficient to characterize the changes of both the elastic and the frictional behavior.

First, we unexpectedly found that for any cell type the $g^{\prime \prime}$ curves from different treatment conditions merged at high frequencies. This indicated that the Newtonian viscosity term $\mu$ was negligible over most of the frequency range studied, and was relatively insensitive to drug treatment. Indeed, the fit of Eq. (3) to the data of a given cell type gave similar values (Table III). $\mu$ did change appreciably in response to cytochalasin $D$ in the case of macrophages and HASM cells (Table III), albeit in different directions. But even in those cell types, the fit of the structural damping equation to the data remained excellent even when we used a single value for $\mu$ that was independent of drug treatment (Fig. 8, Tables I and II).

Second, for any given cell type we unexpectedly found what appeared to be a common intersection, or fixed point, of the $g^{\prime}$ versus frequency curves at a very high frequency, indicating that $g_{0}$ and $\Phi_{0}$ were invariant with different drug treatments (Fig. 5). Statistical analysis of the pooled data in cells with multiple treatments (HASM and neutrophils) revealed that the $g^{\prime}$ curves from multiple treatment conditions indeed could be accounted for with common values for $g_{0}$ and $\Phi_{0}$. The value of $\Phi_{0}$ differed between cell types, but a mixed effect analysis of the unpooled data revealed that the 95\% confidence intervals of the $\Phi_{0}$ estimates were very wide and overlapped between cell types (Table III, Fig. 7). Thus, to a reasonable approximation the data for all cell types could be accounted for with only a single value for $\Phi_{0}$.

The parameter $g_{0}$ differed between cell types, as did the viscosity parameter $\mu$ (Table III). These differences could be a reflection of a number of factors including cell type, specific differences in the attachment properties between cell and bead, different rheological properties between cell types, differences in cell shape, height, cytoskeletal organization, amount of cytoskeletal or crosslinking proteins, activation of motor-proteins, etc. Whatever the origin of the differences between cell types, the contributing factors were cancelled out by our normalization of the $g^{\prime}$ and $g^{\prime \prime}$ data. Normalizing $g^{\prime}$ (measured at $0.75 \mathrm{~Hz}$ ) by $g_{0}$, and normalizing $g^{\prime \prime}$ by $g^{\prime}$ (both measured at $0.75 \mathrm{~Hz}$ ) lead to a collapse of the data based on $x$ alone (Fig. 9): the normalized stiffness $G_{n}$ decreased monotonically, and the normalized friction $\eta$ increased monotonically with increasing $x$. The very same relationships of $G_{n}$ versus $x$ and $\eta$ versus $x$ were found in all cell types that we studied. Those relationships closely follow the predictions from the structural damping equation, with $x$ being the only free parameter.

The observation of universal scaling based on $x$ leads to the prospect of a major empirical simplification. Those relationships suggest that key functions of diverse cytoskeletal proteins, such as their influence on the ability of the cytoskeleton to deform and to flow, may be understood mainly through their ability to modulate $x$. Such behavior is reminiscent of systems that exhibit only a weak dependence of aggregate mechanical behavior on the details of underlying molecular interactions. Familiar examples of such systems include the depression of the freezing point of liquids by addition of arbitrary solutes, or Van't Hoff's law of osmotic pressure. In each of these processes, specific molecular interactions are at some level surely important, but to explain the macroscopic behavior, the molecular details are of only secondary importance. Instead, these systems are colligative in that their macroscopic behavior depends primarily on the number of molecules and their temperature.

The data and analysis presented above establish empirically a series of universal properties that spans diverse cell types. We now turn attention to the question of mechanisms that might underly this behavior. Below we provide a rationale for considering a recently developed theory of soft glassy rheology to explain these findings; this theory derives the structural damping equation from considerations of sta- 
tistical mechanics. The details of this theory have been described elsewhere [7,78,79].

\section{Soft glassy materials}

The class of soft glassy materials (SGM) comprises what would at first glance seem to be a remarkably diverse group of substances that includes foams, pastes, colloids, emulsions, and slurries. Yet, the mechanical behavior of each of these substances is surprisingly alike. The common empirical criteria that define this class of materials are that they are very soft (in the range of $\mathrm{Pa}$ to $\mathrm{kPa}$ ), that both $G^{\prime}$ and $G^{\prime \prime}$ increase with the same weak power-law dependences on frequency, and that the loss tangent $\eta$ is frequency insensitive and of the order $0.1[7,78]$. The data presented here establish that the cells that we studied fulfilled these empirical criteria. The studies described here were not designed to investigate aging phenomena or rejuvenation caused by imposed shear, which would represent even stronger evidence of glassy behavior [80]. Nonetheless, hints of aging, as discussed below, were evident in some of the data.

The physics of glasses in general, and of soft glasses in particular, are much studied but remain poorly understood [81]. In the case of soft glasses, Sollich reasoned that because the materials comprising the class are so diverse, the common rheological features must be not so much a reflection of specific molecules or molecular mechanisms as they are a reflection of generic system properties that play out at some higher level of structural organization [7]. The generic features that all soft glassy materials share are that each is composed of elements that are discrete, numerous and aggregated with one another via weak interactions. In addition, these materials exist far away from thermodynamic equilibrium and are arrayed in a microstructural geometry that is inherently disordered and metastable. All of these features also apply to the CSK of living cells.

Taken together, the cytoskeleton of the living cell satisfies these mechanical criteria as well as the generic features that define the class of soft glassy materials. Accordingly, we propose the working hypothesis that the cytoskeleton of the living cell is a soft glassy material.

To describe the interaction between the elements within the matrix, Sollich developed the theory of soft glassy rheology (SGR) using earlier work by Bouchaud as a point of departure [82]. SGR theory considers that each individual element of the matrix exists within an energy landscape containing many wells, or traps, of differing depth $E$. These traps are formed by interactions of the element with neighboring elements. In the case of living cells those traps might be plausibly thought to be formed by binding energies between neighboring cytoskeletal elements including but not limited to crosslinks between actin filaments, cross-bridges between actin and myosin, hydrophilic interactions between various proteins, charge effects or simple steric constraints.

In Bouchaud's theory of glasses, an element can escape its energy well and fall into another nearby well; such hopping events are activated by thermally driven random fluctuations.
As distinct from Bouchaud's theory, in Sollich's theory of soft glassy materials each energy well is regarded as being so deep that the elements are unlikely to escape the well by thermal fluctuations alone. Instead, elements are imagined to be agitated, or jostled, by their mutual interactions with neighboring elements [7]. A clear notion of the source of the nonthermal agitation remains to be identified, but this agitation can be represented nonetheless by an effective temperature, or noise level, $x$. When $x>1$, there is sufficient agitation in the matrix that the element can hop randomly between wells and, as a result, the system as a whole can flow and become disordered. When $x$ approaches 1 , however, the elements become trapped in deeper and deeper wells from which they are unable to escape-the system exhibits a glass transition and becomes a simple elastic solid with stiffness $G_{0}$.

In SGR theory, the effective noise temperature $x$ is a temperature to the extent that the rate at which elements can hop out of a trap assumes the form $\exp (-E / x)$, where $x$ takes the usual position of a thermal energy $k T$ in the familiar Boltzmann exponential. By analogy, $x$ has been interpreted by Sollich as reflecting jostling of elements by an unidentified but nonthermal origin. This ambiguity has been considered a central limitation of SGR theory [78]. There is no evidence to suggest that the ambiguity surrounding $x$ might be resolved in the case of living cells (as opposed to the inert materials for which SGR theory was originally devised) by an obvious and ready source of non-thermal energy injection, namely, those proteins that go through cyclic conformational changes and thus agitate the matrix by mechanisms that are ATP-dependent.

Remarkably, in the limit that the frequency is small (compared to $\Phi_{0}$ ) and the imposed deformations are small, Sollich's theory leads directly to the structural damping equation [Eq. (3)]. Except for a small discrepancy in some cell types at low frequencies, which we discuss further below, the data reported here establish firmly that the mechanical behavior of cells conform well to Eq. (3) (Table I). If Sollich's quantitative theory and underlying ideas are assumed to apply to the data reported here, then the parameters in Eq. (3) can be identified as follows.

The parameter $x$ is identified as being the noise temperature of the cytoskeletal matrix. The measured values of $x$ in cells lie between 1.15 and 1.35 , indicating that cells exist close to a glass transition.

$G_{0}$ is identified as being the stiffness of the cytoskeleton at the glass transition $(x=1)$. In this connection, Satcher and Dewey [83] developed a static model of cell stiffness based on consideration of cell actin content and matrix geometry. All dynamic interactions were neglected in their model, as would be the case in SGR theory in the limit that $x$ approaches 1 , when all hopping ceases. As such, it might be expected that their model would predict this limiting value of the stiffness, $G_{0}$, as defined in Eq. (3). Indeed, we have found a remarkably good correspondence between their prediction (order of $10 \mathrm{kPa}$ ) and our estimate for $G_{0}(41 \mathrm{kPa}$ in HASM cells).

Finally, $\Phi_{0}$ is identified in Sollich's theory as being the maximum rate at which cytoskeletal elements can escape 
their traps. However, for soft glassy materials in general, and the case of living cells in particular, the factors that determine $\Phi_{0}$ remain unclear. Statistical analysis of our data suggests that $\Phi_{0}$ did not vary with drug treatments and possibly not even across cell type (Fig. 7). But why $\Phi_{0}$ is invariant is not at all clear, and is not explained by SGR theory.

Identification of the living cell in culture as belonging to class of soft glasses at first surprised us, but on further analysis seemed mechanistically plausible. Our data suggest that the living cell exists close to a glass transition and modulates its mechanical properties by moving between glassy states that are "hot," melted and liquidlike, and states that are "cold," frozen and solidlike (Fig. 9). More than a superficial metaphor, this is a precise mechanistic statement of the glass hypothesis and has implications that are quantitative and testable.

The consequences of this hypothesis are interesting. As opposed to focusing attention on the details of specific molecular interactions, the glass hypothesis focuses attention instead on a higher level of structural organization. This is not meant to imply that molecular details are unimportant; to the contrary, there are many individual proteins whose inhibition or dysfunction has catastrophic consequences and whose molecular pathways are critically important. Nonetheless, the existence of master curves (Fig. 9) suggests that molecular interactions may contribute to cytoskeletal dynamics and remodeling mainly to the extent that they are able to modulate the noise temperature $x$. This point of view is novel in that it suggests a generic quality of the contribution of individual molecular species to the noise temperature and associated mechanical behaviors of the CSK. As such, the glass hypothesis to a certain extent subordinates the role of individual molecular species and puts them instead into a deeply integrative context. On the one hand, it implies inherent limits to reductionism, for to study specific molecules in isolation might be to destroy the very interactions that need to be studied. On the other hand, to elucidate a particular molecular-level event in situ without regard to its impact on the noise temperature might be to miss a major facet of its function.

It is interesting that, from a mechanistic point of view, the parameter $x$ plays a central role in the theory of soft glassy materials. At the same time, from a purely empirical point of view, the parameter $x$ is found to play a central organizing role leading to the collapse of all data onto master curves (Fig. 9). Whether or not the measured value of $x$ might ultimately be shown to correspond to a noise temperature, this empirical analysis would appear to provide a unifying framework for studying protein interactions within the complex integrative microenvironment of the cell body.

Departures from structural damping behavior-Hints of aging. In three cell types (human bronchial epithelial cells, macrophages and human neutrophils), we found that the behavior of $g^{\prime \prime}$ departed systematically from the power law behavior implied by Eq. (3); $g^{\prime \prime}$ under baseline conditions became nearly frequency independent below $10 \mathrm{~Hz}$ (Fig. 8). These discrepancies disappeared when the cells were treated with cytochalasin $D$, however.
Closely similar behavior has been reported in soft materials in which the mechanical properties are found to depend on the elapsed time since the sample was prepared [80]. Fielding et al. [80] suggested that this behavior is attributable to aging, wherein elements comprising the matrix are imagined to populate deeper and deeper energy wells as time progresses. The system never achieves a steady state, therefore, and instead behaves as though its longest relaxation time is of the order of its own age. Hence, the longest relaxation time is not a property of any specific molecule of which the system is comprised. Rather, it is a property of the integrated system and its history. SGR theory provides a conceptual framework to study such aging phenomena and predicts aging as an integral part of the low-frequency rheological responses near the glass transition $[7,80]$. The studies reported here were not designed to investigate aging phenomena. Nonetheless, the particular departures of the data from Eq. (3) at low frequencies conform to aging phenomena. We cannot exclude the possibility, however, that the aging-like behavior that we observed was instead attributable to some unspecified relaxation processes occurring at frequencies below our measurement range.

Alternative models. Despite a number of open questions, Sollich's SGR model is simple, intuitive, and predicts structural damping behavior [Eq. (3)]. But soft glassy dynamics as proposed by SGR theory is not the only mechanism that could lead to scale-free mechanical behavior as expressed by structural damping and power-law stress relaxation. Powerlaw behavior could be explained by models containing a large number of viscoelastic compartments with a particular distribution of time constants, but such models lack a mechanistic basis, they require many parameters with no apparent physical meaning, and these parameters would change erratically between different drug treatments of the cell. Powerlaw stress relaxation has been reported for a number of crosslinked polymers at or near the gelation point [84]. However, potential mechanisms leading to this behavior, such as percolation phenomena in self-similar polymer clusters, are still a matter of intense debate [85]. Interestingly, it has been suggested that both the glass transition and critical gelation may arise from a common mechanism, namely kinetic arrest due to crowding of clusters, which is a manifestation of more general jamming transitions [86]. Finally, we cannot rule out the possibility that relaxation processes within the proteins of the cytoskeleton themselves, for instance during folding and unfolding processes and during conformational changes, might account for power-law behavior of the entire cell [87].

\section{CONCLUSIONS}

Time-scale invariance, collapse of data onto master curves, and SGR theory, when taken together, point to metastability of interactions and disorder of the matrix as being generic features that govern integrative mechanical behavior of the cells studied here. It is conceivable that these generic features might transcend cytoskeletal mechanics and carry over into the dynamics of protein interactions whose main manifestation are not at all mechanical, but this possibility is 
not presently amenable to experimental attack. When studying muscle contraction, Hill suggested that the special value of heat release measurements is that they place firm physical limits on underlying mechanical and molecular processes, although they do not point unequivocally to specific molecular events [5]. Similarly, we would say that the special value of the noise temperature is its intimate relation to the mechanical and chemical changes involved, and the sensitivity and speed of the measurement methods that are now available. Measurement of the noise temperature admittedly does not point in a specific way to the individual molecular relaxation processes that occur, but it does provide a firm empirical framework to which those processes seem to conform.

\section{ACKNOWLEDGMENTS}

We thank Stephanie Shore, Paul Moore, Wolfgang Goldmann, and Daniel Tschumperlin for help with cell cultures, Srbolub Mijailovich for providing finite element analysis of cell deformation, Winfried Möller and Maureen Harp for help with bead production, Reynold Panettieri for providing HASM cells, Rick Rogers, Rebecca Sterns, and Marina Puig de Morales for support with microscopy, Peter Malave and Bivash Dasgupta for performing the control experiments on polyacrylamide gels, and C. Forbes Dewey and David Weitz for helpful comments and suggestions. This study was supported by National Institute of Health Grant Nos. HL33009, HL65960, HL59682, and SAF-2002-03616 (Spain).
[1] P. A. Janmey, Physiol. Rev. 78, 763 (1998).

[2] H. Jeong et al., Nature (London) 407, 651 (2000).

[3] E. V. Koonin, Y. I. Wolf, and G. P. Karev, Nature (London) 420, 218 (2002).

[4] Y. L. Kim et al., IEEE J. Sel. Top. Quantum Electron. (to be published).

[5] A. V. Hill, Trails and Trials in Physiology (E. Arnold, London, 1965), pp. 14 and 15.

[6] B. Fabry et al., Phys. Rev. Lett. 87, 148102 (2001).

[7] P. Sollich, Phys. Rev. E 58, 738 (1998).

[8] P. G. de Gennes, Scaling Concepts in Polymer Physics (Cornell University Press, Ithaca, 1979), pp. 134-152.

[9] J. D. Ferry, Viscoelastic Properties of Polymers (Wiley, New York, 1980).

[10] P. A. Valberg and J. P. Butler, Biophys. J. 52, 537 (1987).

[11] N. Wang, J. P. Butler, and D. E. Ingber, Science 260, 1124 (1993).

[12] N. Wang and D. E. Ingber, Biophys. J. 66, 2181 (1994).

[13] G. N. Maksym et al., J. Appl. Physiol. 89, 1619 (2000).

[14] B. Fabry et al., J. Appl. Physiol. 91, 986 (2001).

[15] W. Möller, W. Stahlhofen, and C. Roth, J. Aerosol Sci. 21, S435 (1990).

[16] W. S. Craig et al., Biopolymers 37, 157 (1995).

[17] S. M. Mijailovich et al., J. Appl. Physiol. 93, 1429 (2002).

[18] R. J. Pelham, Jr. and Y. Wang, Proc. Natl. Acad. Sci. U.S.A. 94, 13661 (1997).

[19] T. G. Mason and D. A. Weitz, Phys. Rev. Lett. 74, 1250 (1995).

[20] W. Weber, Ann. Phys. (Leipzig) 54, 1 (1841).

[21] F. Kohlrausch, Ann. Phys. (Leipzig) 128, 1 (1866).

[22] J. Hildebrandt, Bull. Math. Biophys. 31, 651 (1969).

[23] Y. C. Fung, Am. J. Physiol. 213, 1532 (1967).

[24] S. H. Crandall, J. Sound Vib. 11, 3 (1970).

[25] J. J. Fredberg and D. Stamenovic, J. Appl. Physiol. 67, 2408 (1989).

[26] B. Fabry et al., J. Magn. Magn. Mater. 194, 120 (1999).

[27] R. A. Panettieri et al., Am. J. Physiol. 256, C329 (1989).

[28] J. D. Laporte et al., Am. J. Physiol. 275, L491 (1998).

[29] J. L. Coll et al., Proc. Natl. Acad. Sci. U.S.A. 92, 9161 (1995).

[30] P. Ralph and I. Nakoinz, Nature (London) 257, 393 (1975).

[31] J. C. Pinheiro and D. M. Bates, Mixed-Effects Models in S and S-Plus (Springer, New York, 2000).
[32] C. E. Schmidt et al., J. Cell Biol. 123, 977 (1993).

[33] D. Choquet, D. P. Felsenfeld, and M. P. Sheetz, Cell 88, 39 (1997).

[34] P. A. Janmey et al., Nature (London) 345, 89 (1990).

[35] P. A. Janmey et al., J. Biol. Chem. 269, 32503 (1994).

[36] S. Yamada, D. Wirtz, and S. C. Kuo, Biophys. J. 78, 1736 (2000).

[37] M. Yanai et al., Am. J. Physiol. 277, C432 (1999).

[38] C. J. Meyer et al., Nat. Cell Biol. 2, 666 (2000).

[39] J. Chen et al., Am. J. Physiol. Cell Physiol. 280, C1475 (2001).

[40] G. Plopper and D. E. Ingber, Biochem. Biophys. Res. Commun. 193, 571 (1993).

[41] T. Wakatsuki et al., J. Cell. Sci. 114, 1025 (2001).

[42] C. Rotsch et al., Cell Biol. Int. 21, 685 (1997).

[43] R. S. Frank, Blood 76, 2606 (1990).

[44] R. D. Hubmayr et al., Am. J. Physiol. 271, C1660 (1996).

[45] S. A. Shore et al., Am. J. Respir. Cell Mol. Biol. 16, 702 (1997).

[46] N. Wang et al., Am. J. Physiol. Cell Physiol. 282, C606 (2002).

[47] S. S. An et al., Am. J. Physiol. Cell Physiol. 283, C792 (2002).

[48] A. R. Bausch et al., Biophys. J. 75, 2038 (1998).

[49] O. Thoumine and A. Ott, J. Cell. Sci. 110, 2109 (1997).

[50] J. Alcaraz et al., Biophys. J. 84, 2071 (2003).

[51] D. E. Ingber, J. Appl. Physiol. 89, 1663 (2000).

[52] R. E. Buxbaum et al., Science 235, 1511 (1987).

[53] M. Sato, N. Ohshima, and R. M. Nerem, J. Biomech. 29, 461 (1996).

[54] N. Wang and D. E. Ingber, Biochem. Cell Biol. 73, 327 (1995).

[55] N. Wang et al., Am. J. Physiol. 268, C1062 (1995).

[56] F. J. Alenghat et al., Biochem. Biophys. Res. Commun. 277, 93 (2000).

[57] S. G. Shroff, D. R. Saner, and R. Lal, Am. J. Physiol. 269, C286 (1995).

[58] R. E. Mahaffy et al., Phys. Rev. Lett. 85, 880 (2000).

[59] W. H. Goldmann and R. M. Ezzell, Exp. Cell Res. 226, 234 (1996).

[60] I. M. Ward, Mechanical Properties of Solid Polymers (Wiley, Chichester, 1985).

[61] W. Weber, Ann. Phys. (Leipzig) 34, 247 (1835).

[62] J. J. Fredberg et al., J. Appl. Physiol. 74, 1387 (1993). 
[63] J. H. Bates et al., Ann. Biomed. Eng. 22, 674 (1994).

[64] Z. Hantos et al., J. Appl. Physiol. 72, 168 (1992).

[65] G. W. Schmid-Schonbein et al., Biophys. J. 36, 243 (1981).

[66] S. Chien and K. L. Sung, Biophys. J. 46, 383 (1984).

[67] G. I. Zahalak, W. B. McConnaughey, and E. L. Elson, J. Biomech. Eng. 112, 283 (1990).

[68] J. P. Butler and S. M. Kelly, Biorheology 35, 193 (1998).

[69] W. Moller et al., Biophys. J. 79, 720 (2000).

[70] P. A. Valberg and D. F. Albertini, J. Cell Biol. 101, 130 (1985).

[71] A. Yeung and E. Evans, Biophys. J. 56, 139 (1989).

[72] E. Evans and A. Yeung, Biophys. J. 56, 151 (1989).

[73] M. A. Tsai, R. S. Frank, and R. E. Waugh, Biophys. J. 65, 2078 (1993).

[74] O. Thoumine, O. Cardoso, and J. J. Meister, Eur. Biophys. J. 28, 222 (1999)

[75] M. Sato, M. J. Levesque, and R. M. Nerem, Arteriosclerosis (Dallas) 7, 276 (1987).
[76] D. V. Zhelev, D. Needham, and R. M. Hochmuth, Biophys. J. 67, 696 (1994).

[77] T. A. Wilson, J. Appl. Physiol. 77, 1570 (1994).

[78] P. Sollich et al., Phys. Rev. Lett. 78, 2020 (1997).

[79] B. Fabry and J. J. Fredberg, Respir. Physiol. Neurobiol. (to be published).

[80] S. M. Fielding, P. Sollich, and M. E. Cates, J. Rheol. 44, 323 (2000).

[81] S. Torquato, Nature (London) 405, 521 (2000).

[82] J. Bouchaud, J. Phys. I 2, 1705 (1992).

[83] R. L. Satcher, Jr. and C. F. Dewey, Jr., Biophys. J. 71, 109 (1996).

[84] H. H. Winter and F. Chambon, J. Rheol. 30, 367 (1986).

[85] K. Broderix et al., Physica A 302, 279 (2001).

[86] P. N. Segre et al., Phys. Rev. Lett. 86, 6042 (2001).

[87] A. L. Lee and A. J. Wand, Nature (London) 411, 501 (2001). 